\title{
Inverse Backscattering in Two Dimensions *
}

\author{
G. Eskin and J. Ralston \\ Department of Mathematics, UCLA, Los Angeles, CA 90024-1555, USA
}

Received September 26, 1990

\begin{abstract}
This article extends the authors' previous results (Commun. Math. Phys. 124,169-215(1989) to inverse scattering in two space dimensions. The new problem in two dimensions is the behavior of the backscattering amplitude near zero energy. Generically, this has the form

$$
a(\xi /|\xi|,-\xi /|\xi|,|\xi|)=2 \pi(2 \pi \beta+\ln |\xi|)^{-1}+b(\xi),
$$

where $b(0)=0$ and $b(\xi)$ is Hölder continuous. In order to work in weighted Hölder spaces as before, the constant $\beta$ and the function $b(\xi)$ must now be interpreted as "coordinates" on the space of backscattering data. In this setting the mapping to backscattering data is again a local diffeomorphism at a dense open set in the real-valued potentials.
\end{abstract}

\section{Introduction}

This article extends the results of [ER] to problems in two space dimensions. As in [ER] we define the scattering amplitude associated with the Hamiltonian $-\Delta+q(x), x \in \mathbb{R}^{n}$, in the following way. Let $h(\xi, \zeta, k),(\xi, \zeta, k) \in \mathbb{R}^{n} \times \mathbb{R}^{n} \times \mathbb{R}_{+}$, be the solution to the integral equation

$$
h(\xi, \zeta, k)+(2 \pi)^{-n} \int_{\mathbb{R}^{n}} \frac{\hat{q}(\xi-\eta) h(\eta, \zeta, k)}{|\eta|^{2}-(k+i 0)^{2}} d \eta=-\hat{q}(\xi-\zeta),
$$

where $\hat{q}$ denotes the Fourier transform

$$
\hat{q}(\xi)=\int_{\mathbb{R}^{n}} e^{-i x \cdot \xi} q(x) d x .
$$

Then the scattering amplitude is the restriction of $h$ to $|\xi|=|\zeta|=k$, and the backscattering amplitude is $h(\xi,-\xi,|\xi|), \xi \in \mathbb{R}^{n} \backslash 0$. We assume that $\hat{q}$ belongs to one of the weighted Hölder spaces, $H_{\alpha, N}$ used in [ER]. The space $H_{\alpha, N}$ is defined as the closure of $C_{0}^{\infty}\left(\mathbb{R}^{n}\right)$ in the norm

$$
\|f\|_{\alpha, N}=\left\|\Lambda^{N} f\right\|_{\alpha}
$$

\footnotetext{
* Partially supported by NSF Grant DMS89-02246
} 
where \|\|$_{\alpha}$ is the Hölder $\alpha$-norm and $\Lambda(\xi)=\left(1+|\xi|^{2}\right)^{1 / 2}$. The function $h(\xi, \zeta, k)$ is understood to be the solution of (I.1) belonging to $H_{\alpha, N}$ in $\xi$ for each value of the parameters $(\zeta, k)$.

In [ER] we showed that in three dimensions on an open set of $\hat{q}$ the mapping from $\hat{q}$ to backscattering is locally an analytic isomorphism of $H_{\alpha, N}$. The impossibility of obtaining this result in two dimensions is due to the singularity in the fundamental solution for $-\Delta-(k+i 0)^{2}$ at $k=0$. While in three dimensions one has

$$
\begin{aligned}
E_{k}(x) & =(2 \pi)^{-3} \int_{\mathbb{R}^{3}} \frac{e^{i x \cdot \eta}}{|\eta|^{2}-(k+i 0)^{2}} d \eta \\
& =(4 \pi)^{-1}|x|^{-1} e^{i k|x|}
\end{aligned}
$$

in two dimensions

$$
\begin{aligned}
E_{k}(x) & =(2 \pi)^{-2} \int_{\mathbb{R}^{2}} \frac{e^{i x \cdot \eta}}{|\eta|^{2}-(k+i 0)^{2}} d \eta=\frac{i}{4} H_{0}^{(1)}(k|x|) \\
& =\frac{i}{4}\left(J_{0}(k|x|)+i Y_{0}(k|x|)\right),
\end{aligned}
$$

and $Y_{0}(k|x|) \sim \frac{2}{\pi} \ln k$ as $k \rightarrow 0$. This singularity produces the following generic behavior for $h(\xi,-\xi,|\xi|)$ as $\xi \rightarrow 0$ (see Theorem 2.4): for $\hat{q} \in H_{\alpha, N}, N>0,0<\alpha<1$,

$$
h(\xi,-\xi,|\xi|)=2 \pi(\ln |\xi|+2 \pi \beta)^{-1}+b_{1}(\xi)
$$

where $b_{1}(0)=0$ and $b_{1}$ is Hölder $\alpha$-continuous. Note that

$$
\beta=\lim _{\xi \rightarrow 0}\left(\frac{\ln |\xi|}{2 \pi}\right)^{2}\left(\frac{2 \pi}{\ln |\xi|}-h(\xi,-\xi,|\xi|)\right) .
$$

Hence, fixing $\chi \in C_{0}^{\infty}\left(\mathbb{R}^{2}\right)$ with $\chi \equiv 1$ on a neighborhood of $\xi=0$, we can define "coordinates" on the backscattering data $\left(\beta, b_{0}\right) \in \mathbb{C} \times\left\{f \in H_{\alpha, N}: f(0)=0\right\}$ with $\beta$ defined by (I.3) and

$$
b_{0}(\xi)=(1-\chi(\xi)) h(\xi,-\xi,|\xi|)+\chi(\xi)\left(h(\xi,-\xi,|\xi|)-2 \pi(\ln |\xi|+2 \pi \beta)^{-1}\right) .
$$

Then we show that for $0<\alpha<\frac{1}{2}$ and $N>0$,

$$
S: \hat{q} \rightarrow \frac{1}{\beta} \chi(\xi)+b_{0}(\xi)
$$

is a local analytic isomoprhism of $H_{\alpha, N}$ on an open subset of $H_{\alpha, N}$. This subset has dense intersection with $H_{\alpha, N}^{r}=\left\{\hat{q} \in H_{\alpha, N}: \hat{q}(-\xi)=\overline{\hat{q}}(\xi)\right\}$, the subspace of $H_{\alpha, N}$ over the reals consisting of Fourier transforms of real-valued potentials. As in [ER] the "restricted" mapping $S^{r}: H_{\alpha, N}^{r} \rightarrow H_{\alpha, N}^{r}$, taking $\hat{q}$ to the projection of $S(\hat{q})$ onto $H_{\alpha, N}^{r}$, has a Fredholm Frechet derivative of index zero on a dense open subset $\mathcal{O}$ of $H_{\alpha, N}^{r}$. Thus, by the argument from real analyticity used in [ER], the restricted backscattering mapping $S^{r}$, is a local isomorphism on a dense open subset of each connected component of $\mathcal{O}$ which contains a $\hat{q}_{0}$ such that $d S^{r}\left(\hat{q}_{0}\right)$ is invertible.

Many of the arguments needed to establish these results are identical to those 
in [ER]. We will omit these here and restrict attention to the new problems which arise in two dimensions.

There is a very extensive literature on the problem of determining the set of full scattering amplitudes in $\mathbb{R}^{n}$, the functions $h(k \theta, k \omega, k)$ on $S^{n-1} \times S^{n-1} \times \mathbb{R}_{+}$in our notation, see Faddeev [F], Newton [N], Beals-Coifman [BS], NachmanAblowitz [NA], Novikov-Khenkin [NK], Melin [M], and Weder [W]. These works deal with $n \geqq 3$. For $n=2$ results on the problem of characterizing the set of scattering amplitudes have been obtained by Cheney [C]. Much less work has been done on the problem of determining potentials from backscattering amplitudes. Aside from the formal results of Prosser [P] and the numerical study by Bayliss, Li and Morawetz, [BLM], we only know of [ER] and the recent work of Melrose and Uhlmann, [ML]. These papers deal with $n=3$ in [P] and [ER], general odd $n \geqq 3$ in [MU], and $n \geqq 1$ in [BLM].

We will use the notation of [ER]. In particular

and

$$
\begin{aligned}
\Delta(\mu) f(\xi, \zeta, k) & =f(\xi+\mu, \zeta, k)-f(\xi, \zeta, k), \\
\Delta(v) f(\xi, \zeta, k) & =f(\xi, \zeta+v, k)-f(\xi, \zeta, k), \\
\Delta(s) f(\xi, \zeta, k) & =f(\xi, \zeta, k+s)-f(\xi, \zeta, k) \quad \text { with } \quad s>0,
\end{aligned}
$$

$$
f_{\zeta}(\xi)=f(\xi-\zeta)
$$

\section{Direct Estimates}

We begin with the analogue of Theorem 2.1 of [ER]. Let $\chi$ and $\chi_{0}$ be functions in $C_{0}^{\infty}\left(\mathbb{R}^{2}\right)$ and $C_{0}^{\infty}(\mathbb{R})$ respectively satisfying $\chi(\xi)=1$ for $|\xi|<\rho, \chi(\xi)=0$ for $|\xi|>2 \rho, \chi_{0}(k)=1$ for $|k|<\rho_{0}$ and $\chi_{0}(k)=0$ for $|k|>2 \rho_{0}$. We define

$$
\left[A_{1}(\hat{q}, k) f\right](\xi)=(2 \pi)^{-2} \int_{\mathbb{R}^{2}} \frac{\hat{q}(\xi-\eta)\left(f(\eta)-f(0) \chi(\eta) \chi_{0}(k)\right)}{|\eta|^{2}-(k+i 0)^{2}} d \eta .
$$

Theorem 1.1. The operator $A_{1}$ satisfies

$$
\left\|\Lambda_{\zeta}^{N} A_{1}(\hat{q}, k) \Lambda_{\zeta}^{-N} \Lambda^{\delta} f\right\|_{\alpha} \leqq C(1+k)^{-\gamma}\|\hat{q}\|_{\alpha, N}\|f\|_{\alpha-\varepsilon},
$$

where $\Lambda_{\zeta}(\xi)=\Lambda(\xi-\zeta), \quad 0<\alpha<1, \quad N>0, \quad 0 \leqq \varepsilon<\alpha, \quad 0 \leqq \delta<\min \{1, N\}$ and $\gamma<\min \{1-\delta, N-\delta\}$.

Remark. The statement given here is merely a way of combining what are really two results. When $k \in\left[2 \rho_{0}, \infty\right), A_{1}(\hat{q}, k)$ reduces to what was called $A(\hat{q}, k)$ in [ER] and the proof of Theorem 1.1 is precisely the same as the proof of Theorem 2.1 in $[E R]$.

To prove Theorem 1.1 we will need the following lemmas (cf. [ER], Lemmas 2.2 and 2.3).

Lemma 1.2. Let

$$
I(k, \xi)=\int_{0}^{2 \pi}\left(1+|\xi-k \omega|^{2}\right)^{-N / 2} d \theta,
$$

where $\omega=(\cos \theta, \sin \theta), \xi \in \mathbb{R}^{2}$ and $k \in \mathbb{R}_{+}$. 
Then

$$
I(k, \xi) \leqq \begin{cases}C k^{-1} & \text { for } \quad N>1 \\ C k^{-1} \ln (1+k) & \text { for } \quad N=1 \\ C k^{-N} & \text { for } 0<N<1\end{cases}
$$

where $C$ is independent of $\xi$ and $k$.

Proof. We have

$$
I=\int_{-\pi}^{\pi}\left(1+k^{2}+|\xi|^{2}-2 k|\xi| \cos \theta\right)^{-N / 2} d \theta
$$

and for any $\delta>0$,

$$
\int_{\delta<|\theta| \leqq \pi}\left(1+k^{2}+|\xi|^{2}-2 k|\xi| \cos \theta\right)^{-N / 2} d \theta \leqq C_{\delta}\left(1+k^{2}\right)^{-N / 2} .
$$

Furthermore, for $\delta$ small enough that $\cos \theta<1-\frac{\theta^{2}}{4}$ for $|\theta|<\delta$,

$$
\int_{|\theta|<\delta}\left(1+k^{2}+|\xi|^{2}-2 k|\xi| \cos \theta\right)^{-N / 2} d \theta \leqq \int_{|\theta|<\delta}\left(1+(k-|\xi|)^{2}+\frac{k|\xi| \theta^{2}}{2}\right)^{-N / 2} d \theta
$$

Making the change of variables $t=(k|\xi|)^{1 / 2} \theta$,

$$
\int_{|\theta|<\delta}\left(1+(k-|\xi|)^{2}+\frac{1}{2} k|\xi| \theta^{2}\right)^{-N / 2} d \theta \leqq(k|\xi|)^{-1 / 2} \int_{|t|<(k|\xi|)^{1 / 2} \delta}\left(1+t^{2} / 2\right)^{-N / 2} d t .
$$

If $|k-| \xi||<\frac{1}{2} k$, this leads directly to (1.2). However, if $|k-| \xi||>\frac{1}{2} k$,

$$
\int_{|\theta|<\delta}\left(1+(k-|\xi|)^{2}+\frac{1}{2} k|\xi| \theta^{2}\right)^{-N / 2} d \theta<C\left(1+k^{2}\right)^{-N / 2} \text {. }
$$

Lemma 1.3. Let

$$
J(k, \xi, \zeta)=\int_{|| \eta|-k|>1} \frac{\Lambda_{\zeta}^{N}(\xi) \Lambda^{\delta}(\eta)}{\Lambda_{\xi}^{N}(\eta) \Lambda_{\zeta}^{N}(\eta)} \frac{d \eta}{\left.|| \eta\right|^{2}-k^{2} \mid},
$$

where $0<\delta<\min \{1, N\}$. Then for $0<\gamma<\min \{N-\delta, 1-\delta\}$ we have

$$
J(k, \xi, \zeta) \leqq C(1+k)^{-\gamma} .
$$

Proof. We have

$$
\begin{aligned}
J(k, \xi, \zeta) & \leqq C \int_{|| \eta|-k|>1}\left(\frac{1}{\Lambda_{\xi}^{N}(\eta)}+\frac{1}{\Lambda_{\zeta}^{N}(\eta)}\right) \frac{\Lambda^{\delta}(\eta)}{\left.|| \eta\right|^{2}-k^{2} \mid} d \eta \\
& \leqq C \int_{|| \eta|-k|>1} \frac{\Lambda^{\delta-\beta}(\eta)}{\left.|| \eta\right|^{2}-k^{2} \mid}|\eta| d|\eta|
\end{aligned}
$$

by Lemma 1.2 , where $\beta=1$ for $N>1$, and $\beta=\gamma+\delta+\frac{1}{2}(N-\gamma-\delta)$ for $N \leqq 1$. Since $\delta-\beta<0$, for $k<\frac{1}{2}$ we have $J<C$. For $k>\frac{1}{2}$, we set $|\eta|=k t$ and have

$$
\begin{aligned}
J(k, \xi, \zeta) & \leqq C \int_{|t-1|>1 / k}\left(1+k^{2} t^{2}\right)^{(\delta-\beta) / 2} \frac{t}{\left|t^{2}-1\right|} d t \\
& \leqq C \int_{|t-1|>1 / k} \frac{d t}{|t-1||k t|^{\beta-\delta}} \leqq C k^{\delta-\beta} \ln k .
\end{aligned}
$$


The proof of Theorem 1.1 will use the decompositions of integrals that were used in the proof of [ER, Theorem 2.1] with the same labels, $I_{1}, I_{2}, I_{3}$ etc. One sees that the only new features here are that $d \eta=|\eta| d|\eta| d \theta$ instead of $d \eta=|\eta|^{2} d|\eta| d \Omega$ and that we can use $|P f(\eta)|=|f(\eta)-f(0) \chi(\eta)| \leqq C|\eta|^{\alpha}\|f\|_{\alpha}$ in place of $|f(\eta)| \leqq\|f\|_{\alpha}$.

In two dimensions formula 2.6 of [ER] becomes, for $0<k<1$,

$$
\lim _{\varepsilon \downarrow 0} \int_{|| \eta|-k|<1} \frac{|\eta| d|\eta|}{|\eta|^{2}-(k+i \varepsilon)^{2}}=\left.\frac{1}{2} \ln \left(|\eta|^{2}-(k+i \varepsilon)^{2}\right)\right|_{0} ^{k+1}=\frac{1}{2} \ln (1+2 k)-\ln k-\frac{\pi i}{2} .
$$

Since Lemma 1.2 implies

$$
\begin{aligned}
& \left|\int_{0}^{2 \pi} \frac{\Lambda_{\zeta}^{N}(\xi) \Lambda_{\xi}^{N}(k \omega) \hat{q}(\xi-k \omega)\left(f(k \omega)-f(0) \chi(k \omega) \chi_{0}(k)\right)}{\Lambda_{\zeta}^{N}(k \omega) \Lambda_{\xi}^{N}(k \omega)} d \theta\right| \\
& \quad \leqq \frac{C \operatorname{Min}\left\{k^{\alpha}, 1\right\}}{(1+k)^{\gamma+\delta}}\|q\|_{\alpha, N}\|f\|_{\alpha},
\end{aligned}
$$

we again have $\left|I_{3}\right| \leqq C(1+k)^{-\gamma}\|q\|_{\alpha, N}\|f\|_{\alpha}$. Since this estimate holds if we apply $|\mu|^{-\alpha} \Delta(\mu)$ to the integral as well, we conclude that $I_{3}$ satisfies the required estimate.

The terms $I_{1}$ and $|\mu|^{-\alpha} \Delta(\mu) I_{1}$, are both bounded by

$$
C \int_{0}^{\infty}(1+|\eta|)^{\delta-\beta-1} d|\eta|\|\hat{q}\|_{\alpha, N}\|f\|_{0},
$$

where $\beta=1$ for $N>1$ and $\beta=\gamma+\delta+\frac{1}{2}(N-\gamma-\delta)<N$ for $0<N \leqq 1$. Moreover,

$$
\begin{aligned}
\left|I_{2}\right| & \leqq C \int_{|| \eta|-k|<1} \frac{\left(1+|\eta|^{2}\right)^{(\delta-\beta) / 2}|\eta| d|\eta|}{(|\eta|+k)|| \eta|-k|^{1-\alpha}}\|\hat{q}\|_{\alpha, N}\|f\|_{\alpha} \\
& \leqq(1+|k|)^{\delta-\beta}\|\hat{q}\|_{\alpha, N}\|f\|_{\alpha} .
\end{aligned}
$$

There is no change in the estimate of $\left|\Delta(\mu) J_{1}\right|$, except that $\delta-\beta+1$ becomes $\delta-\beta$. This is also true of $K_{1}, K_{4}, L_{1}, L_{2}, L_{3}, L_{4}$ and $M$. The estimate of the integral $P$ for $0<k<1$ is now

$$
|P| \leqq C k^{\alpha}|\mu|^{\alpha}\|\hat{q}\|_{\alpha, N}\|f\|_{\alpha},
$$

and we have

$$
\begin{aligned}
\int_{1>|| \eta|-k|>2|\mu|} \frac{|\eta| d|\eta|}{|\eta|^{2}-k^{2}}= & \frac{1}{2} \int_{1>|| \eta|-k|>2|\mu|} \frac{d|\eta|}{|\eta|+k} \\
& +\frac{1}{2} \int_{1>|| \eta|-k|>2|\mu|} \frac{1}{|\eta|-k} d|\eta| .
\end{aligned}
$$

These integrals are bounded by $\ln k$ independently of $\mu$. Since $k^{\alpha} \ln k$ is bounded when $0<k<1$, we conclude that $M_{2}$ satisfies the desired estimate. For $M_{3}$ we have for $0<k<1$,

$$
\begin{aligned}
\left|M_{3}\right| & \leqq C k^{\alpha}\|\hat{q}\|_{\alpha, N}\|f\|_{\alpha} \int_{1>|| \eta|-k|>2|\mu|} \frac{|\eta|\left(|\mu|^{\alpha}|| \eta|-k|^{\alpha}+|\mu|^{2 \alpha}\right)}{\left.|| \eta\right|^{2}-k^{2} \mid(|\eta|+|\mu|)^{\alpha}} d|\eta| \\
& \leqq C\|\hat{q}\|_{\alpha, N}\|f\|_{\alpha} \int_{1>|| \eta|-k|>2|\mu|} \frac{|\mu|^{\alpha}|| \eta|-k|^{\alpha}+|\mu|^{2 \alpha}}{|| \eta|-k|} d|\eta|
\end{aligned}
$$

which also leads to the desired estimate. 

2.2].

The function $A_{1}(\hat{q}, k) f$ also has the regularity in $k$ described in [ER, Theorem

Theorem 1.4. For $N, \delta, \alpha$ in the set used in Theorem 1.1, $s>0$ and $f$ satisfying $f(0, \xi, \zeta)=0$, we have for $k<\rho_{0}$,

$$
\sup _{\xi, \zeta} \Lambda_{\zeta}^{N}(\xi)\left|\int_{\mathbb{R}^{2}} f(\eta, \xi, \zeta)\left(\Delta(s) \frac{1}{|\eta|^{2}-(k+i 0)^{2}}\right) d \eta\right| \leqq s^{\alpha} \sup _{\xi, \zeta}\left\|\Lambda^{-\delta} \Lambda_{\zeta}^{N} \Lambda_{\zeta}^{N} f\right\|_{\alpha} .
$$

The proof of Theorem 1.4 follows the proof of [ER, Theorem 2.2] in the same manner that the proof of Theorem 1.3 followed the proof of Theorem 2.1 of [ER], and we omit it.

Much of the analysis of Sect. 3 of [ER] applies to $A_{1}(\hat{q}, k)$ in two dimensions. In particular, there is an open set $\mathcal{O}_{0}$ of $\hat{q}$ in $H_{\alpha, N}$ (which will be discussed in Sect. 3) such that for $\hat{q} \in \mathcal{O}_{0}$ the operator

$$
I+\Lambda_{\zeta}^{N} A_{1}(\hat{q}, k) \Lambda_{\zeta}^{-N}
$$

is invertible on $C^{\alpha}\left(\mathbb{R}^{2}\right)$ for $(\zeta, k) \in \mathbb{R}^{2} \times\left[0, \rho_{0}\right]$ and the norm of its inverse is uniformly bounded for $(\zeta, k) \in \mathbb{R}^{2} \times\left[0, \rho_{0}\right]$. Thus for $\hat{q} \in \mathcal{O}_{0}$ the solution, $h_{1}(\xi, \zeta, k)$, of

$$
h_{1}+A_{1}(\hat{q}, k) h_{1}=-\hat{q}_{\zeta}
$$

exists for $(\zeta, k) \in \mathbb{R}^{2} \times\left[0, \rho_{0}\right]$ and satisfies

$$
\left\|\Lambda_{\zeta}^{N} h_{1}(\cdot, \zeta, k)\right\|_{\alpha}<C\|\hat{q}\|_{\alpha, N}
$$

with $C$ independent of $(\zeta, k)$.

The function $h_{1}(\xi, \zeta, k)$ is not uniformly $\alpha$-Hölder continuous in $\zeta$ and $k$ near $k=0$. In the following paragraphs, assuming $k<\rho_{0}<1$ we will derive estimates for $\Delta(v) h_{1}$ and $\Delta(s) h_{1}$ as well as $\Delta(v) P h_{1}$ and $\Delta(s) P h_{1}$, where

$$
[P f](\xi)=f(\xi)-f(0) \chi(\xi) .
$$

These estimates will be $\alpha$-Hölder estimates with weights of $|\ln k|$. The methods we use to derive them are those of Sect. 3 of [ER]. We will use the density of $C_{0}^{\infty}$ in $H_{\alpha, N}$ to write $\hat{q}=\hat{q}_{1}+\hat{q}_{\infty}$, where $\left\|\bar{q}_{1}\right\|_{\alpha, N}<\varepsilon_{0}$ and $\hat{q}_{\infty} \in C_{0}^{\infty}\left(\mathbb{R}^{2}\right)$. The constant $\varepsilon_{0}$ is chosen small enough that the Neumann series for $\left(I+A_{1}\left(\hat{q}_{1}, k\right)\right)^{-1}$ converges. Hence

and

$$
h_{1}+A_{1}\left(\hat{q}_{1}, k\right) h_{1}+A_{1}\left(\hat{q}_{\infty}, k\right) h_{1}=-\hat{q}_{\zeta}
$$

$$
h_{1}=\left(I+A_{1}\left(\hat{q}_{1}, k\right)\right)^{-1}\left(g_{1}-\hat{q}_{\zeta}\right)
$$

where $g_{1}$ is the solution to

$$
g_{1}+A_{1}\left(\hat{q}_{\infty}, k\right)\left(I+A_{1}\left(\hat{q}_{1}, k\right)\right)^{-1} g_{1}=A_{1}\left(\hat{q}_{\infty}, k\right)\left(I+A_{1}\left(\hat{q}_{1}, k\right)\right)^{-1} \hat{q}_{\zeta} .
$$

The general term in the Neumann series expansion of $\left(I+A_{1}\left(\hat{q}_{1}, k\right)\right)^{-1} q_{\zeta}$ has the form (suppressing a factor of $(-1)^{n}(2 \pi)^{-2 n}$ ):

$$
\begin{aligned}
& {\left[\left(A_{1}\left(\hat{q}_{1}, k\right)\right)^{n} \hat{q}_{\zeta}\right](\xi)=\int_{\mathbb{R}^{2 n}} \hat{q}_{1}\left(\xi-\eta_{n}\right)} \\
& \frac{\left(\prod_{i=2}^{n}\left(\hat{q}_{1}\left(\eta_{i}-\eta_{i-1}\right)-\hat{q}_{1}\left(-\eta_{i-1}\right) \chi\left(\eta_{i}\right)\right)\right)\left(\hat{q}\left(\eta_{1}-\zeta\right)-\hat{q}(-\zeta) \chi\left(\eta_{1}\right)\right) d \eta_{1} \cdots d \eta_{n}}{\prod_{i=1}^{n}\left(\left|\eta_{i}\right|^{2}-(k+i 0)^{2}\right)}
\end{aligned}
$$


for $n \geqq 1$. Introducing

we have

$$
\left[A_{1}^{t}(p, k) g\right](\xi)=(2 \pi)^{-2} \int_{\mathbb{R}^{2}} g(\eta) \frac{(p(\eta-\xi)-p(-\xi) \chi(\eta))}{|\eta|^{2}-(k+i 0)^{2}} d \eta
$$

$$
\left[\left(A_{1}\left(\hat{q}_{1}, k\right)\right)^{n} \hat{q}_{\zeta}\right](\xi)=\left[A_{1}^{t}(\hat{q}, k)\left(A_{1}^{t}\left(\hat{q}_{1}, k\right)\right)^{n-1} f\right](\zeta),
$$

where $f(\xi, \eta)=\hat{q}_{1}(\xi-\eta)$. We will show that for $0<k<\rho_{0}<1$,

a) $\left\|A_{1}^{t}(p, k) g\right\|_{\alpha^{\prime}, N} \leqq C\|p\|_{\alpha, N}\|g\|_{\alpha^{\prime}, N}$

and

b) $\left\|A_{1}^{t}(p, k) g\right\|_{\alpha, N} \leqq C\|p\|_{\alpha, N}\|g\|_{\alpha^{\prime}, N}|\ln k|$

for $0<\alpha^{\prime}<\alpha$. Then it will follow that

$$
\left|\Lambda_{\zeta}^{N}(\xi) \Delta(v)\left[\left(A_{1}\left(\hat{q}_{1}, k\right)\right)^{n} \hat{q}_{\zeta}\right](\xi)\right| \leqq C^{n}\left\{\left.v\right|^{\alpha}\|\hat{q}\|_{\alpha, N}\left\|\hat{q}_{1}\right\|_{\alpha, N}^{n}\{\ln k\}\right.
$$

uniformly in $\xi$. Moreover, using Theorem 1.4 and Liebnitz's expansion, a) and b) also imply that

$$
\left|\Lambda_{\zeta}^{N}(\xi) \Delta(s)\left[\left(A_{1}\left(\hat{q}_{1}, k\right)\right)^{n} \hat{q}_{\zeta}\right](\xi)\right| \leqq n C^{n} s^{\alpha}\|\hat{q}\|_{\alpha, N}\left\|q_{1}\right\|_{\alpha, N}^{N}|\ln k|
$$

uniformly in $\xi$.

The estimate a) is an immediate consequence of Theorem 1.1. Let

$$
f(\eta, \xi, \mu)=\frac{\Delta(\mu)(p(\eta-\xi)-p(-\xi) \chi(\eta))}{|\mu|^{\alpha^{\prime}}} .
$$

Then $\left\|f(\cdot, \xi, \mu) \Lambda_{\xi}^{N}\right\|_{\alpha-\alpha^{\prime}} \leqq\|p\|_{\alpha, N}$. Since $f(0, \xi, \mu)=0$,

$$
\left[|\mu|^{-\alpha^{\prime}} \Delta(\mu)\left(A_{1}^{t}(p, k) g\right)\right](\xi)=\left[A_{1}(\hat{q}, k) f\right](0)
$$

where $q(\eta)=g(-\eta)$. Thus a) follows from Theorem 1.1 with $\zeta=\xi$.

To prove b) we need to review the proof of Theorem 1.1. Using the decomposition

$$
\Lambda_{\zeta}^{N} A_{1}^{t}(p, k) \Lambda_{\zeta}^{-N} g=I_{1}+I_{2}+I_{3}
$$

as in the proof of Theorem 2.1 of [ER], we have for $0<k<\rho_{0}<1$,

$$
I_{3}=\left(-\ln k+\frac{1}{2} \ln (1+2 k)+\frac{\pi i}{2}\right) \int_{S_{1}} \frac{\Lambda_{\zeta}^{N}(\xi)}{\Lambda_{\zeta}^{N}(k \omega)} g(k \omega)(p(k \omega-\xi)-p(-\xi) \chi(k \omega)) d \omega .
$$

Since $|p(k \omega-\xi)-p(-\xi)|<c k^{\alpha} \Lambda^{-N}(\xi)$, this implies

$$
\left|I_{3}\right| \leqq C\|g\|_{0}\|p\|_{\alpha, N},
$$

as before. However, when we apply $\Delta(\mu)$ to $I_{3}$ there are no useful cancellations between $\Delta(\mu) p(k \omega-\xi)$ and $\Delta(\mu) p(-\xi)$ as $k \rightarrow 0$. Thus we only have

$$
\left|\Delta(\mu) I_{3}\right| \leqq C\|g\|_{0}\|p\|_{\alpha, N}|\mu|^{\alpha}(|\ln k|+1) .
$$

As in the proof of Theorem 1.1, all other estimates from the proof of Theorem 2.1 [ER] go through mutatis mutandis until one reaches $M_{2}$. In $M_{2}$ we no longer have the factor of $k^{\alpha}$ so that now

$$
\left|M_{2}\right| \leqq C|\mu|^{\alpha}\|p\|_{\alpha, N}\|g\|_{\alpha}(|\ln k|+1) .
$$


For $M_{3}$ we now have

$$
\left|M_{3}\right| \leqq C\|p\|_{\alpha, N}\|g\|_{\alpha} \int_{2|\mu|<|| \eta|-k|<1} \frac{|\eta|}{\left.|| \eta\right|^{2}-k^{2} \mid}\left(\frac{|\mu|^{\alpha}|| \eta|-k|^{\alpha}+|\mu|^{2 \alpha}}{(|\eta|+|\mu|)^{\alpha}}\right) d|\eta| .
$$

For $2|\mu|<k<\mid$ this gives

$$
\begin{aligned}
\left|M_{3}\right| \leqq & C\|p\|_{\alpha, N}\|g\|_{\alpha}|\mu|^{\alpha}\left[\int_{k+2|\mu|}^{1+k} \frac{|\eta|^{1-\alpha}\left(|| \eta|-k|^{\alpha}+|\mu|^{\alpha}\right)}{|\eta|^{2}-k^{2}} d|\eta|\right. \\
& \left.+\int_{0}^{k-2|\mu|} \frac{|\eta|^{1-\alpha}\left(|| \eta|-k|^{\alpha}+|\mu|^{\alpha}\right)}{k^{2}-|\eta|^{2}} d|\eta|\right] \\
= & C\|p\|_{\alpha, N}\|g\|_{\alpha}|\mu|^{\alpha}\left[\int_{1+2|\mu| / k}^{1+1 / k} \frac{s^{1-\alpha}\left(|s-1|^{\alpha}+|\mu|^{\alpha} / k^{\alpha}\right)}{s^{2}-1} d s\right. \\
& \left.+\int_{0}^{1-2|\mu| / k} \frac{s^{1-\alpha}\left(|s-1|^{\alpha}+|\mu|^{\alpha} / k^{\alpha}\right)}{1-s^{2}} d s\right] \\
\leqq & C\|p\|_{\alpha, N}\|g\|_{\alpha}|\mu|^{\alpha}\left(1+|\ln k|+\frac{|\mu|^{\alpha}}{k^{\alpha}}\left|\ln \frac{|\mu|}{k}\right|\right) \\
\leqq & C\|p\|_{\alpha, N}\|g\|_{\alpha}|\mu|^{\alpha}(1+|\ln k|) .
\end{aligned}
$$

For $k<2|\mu|<1,(1.6)$ gives

$$
\begin{aligned}
\left|M_{3}\right| & \leqq C\|p\|_{\alpha, N}\|g\|_{\alpha}|\mu|^{\alpha} \int_{k+2|\mu|}^{1} \frac{|| \eta|-k|^{\alpha}+|\mu|^{\alpha}}{|| \eta|-k||| \eta|+| \mu||^{\alpha}} d|\eta| \\
& \leqq C\|p\|_{\alpha, N}\|g\|_{\alpha}|\mu|^{\alpha} \int_{k+2|\mu|}^{1} \frac{d|\eta|}{|| \eta|-k|},
\end{aligned}
$$

since $|\eta|+|\mu|>\frac{1}{2}|| \eta|-k|$. Thus

$$
\begin{aligned}
\left|M_{3}\right| & \leqq C\|p\|_{\alpha, N}\|g\|_{\alpha}|\mu|^{\alpha}(|\ln | \mu||+1) \\
& \leqq C\|p\|_{\alpha, N}\|g\|_{\alpha}|\mu|^{\alpha}(|\ln k|+1),
\end{aligned}
$$

and we have established $b$ ) in the case $\alpha^{\prime}=\alpha$. However, this suffices for the general case just as in the proof of Theorem 1.1 (see [ER, p. 175]). This completes the proof of (1.4) and (1.5).

To estimate $P\left(I+A_{1}\left(\hat{q}_{1}, k\right)\right)^{-1} \hat{q}_{\zeta}$ we note that

$$
\left[P\left(A_{1}\left(\hat{q}_{1}, k\right)\right)^{n} \hat{q}_{\zeta}\right](\xi)=\left[A_{1}^{t}(\hat{q}, k)\left(A_{1}^{t}\left(\hat{q}_{1}, k\right)\right)^{n-1} f\right](\zeta)
$$

with $f(\xi, \eta)=\hat{q}_{1}(\xi-\eta)-\hat{q}_{1}(-\eta) \chi(\xi)$. Thus

$$
\left\|\Lambda_{\xi}^{N} f\right\|_{\alpha^{\prime}} \leqq C|\xi|^{\alpha-\alpha^{\prime}}\left\|\hat{q}_{1}\right\|_{\alpha, N}
$$

and a) and b) imply that for $n \geqq 1,0<k<\rho_{0}<1$ and $0<\alpha^{\prime}<\alpha$,

$$
\left|\Lambda_{\zeta}^{N}(\xi) \Delta(v)\left[P\left(A_{1}\left(\hat{q}_{1}, k\right)\right)^{n} \hat{q}_{\zeta}\right](\xi)\right| \leqq C^{n}|v|^{\alpha}\|\hat{q}\|_{\alpha, N}\left\|\hat{q}_{1}\right\|_{\alpha, N}^{n}|\xi|^{\alpha-\alpha^{\prime}}|\ln k|
$$

and

$$
\left.\mid \Lambda_{\zeta}^{N} \Delta(s)\left[P A_{1}\left(\hat{q}_{1}, k\right)\right)^{n} \hat{q}_{\zeta}\right]\left.(\xi)\left|\leqq n C^{n} S^{\alpha}\|\hat{q}\|_{\alpha, N}\left\|\hat{q}_{1}\right\|_{\alpha, N}^{n}\right| \xi\right|^{\alpha-\alpha^{\prime}}|\ln k| .
$$

Finally, taking $\left\|\hat{q}_{1}\right\|$ sufficiently small, i.e. $\varepsilon_{0}$ sufficiently small, (1.4), (1.5), (1.7) and (1.8) imply 


$$
\begin{aligned}
\left|\Lambda_{\zeta}^{N} \Delta(v)\left(I+A_{1}\left(\hat{q}_{1}, k\right)\right)^{-1} \hat{q}_{\zeta}\right| & \leqq C|v|^{\alpha}\|\hat{q}\|_{\alpha, N}|\ln k| \\
\left|\Lambda_{\zeta}^{N} \Delta(s)\left(I+A_{1}\left(\hat{q}_{1}, k\right)\right)^{-1} q_{\zeta}\right| & \leqq C s^{\alpha}\|\hat{q}\|_{\alpha, N}|\ln k| \\
\left|\Lambda_{\zeta}^{N} \Delta(v) P\left(I+A_{1}\left(\hat{q}_{1}, k\right)\right)^{-1} \hat{q}_{\zeta}\right| & \leqq C|v|^{\alpha}\|\hat{q}\|_{\alpha, N}\left(1+|\ln k||\xi|^{\alpha-\alpha^{\prime}}\right), \\
\left|\Lambda_{\zeta}^{N} \Delta(s) P\left(I+A_{1}\left(q_{1}, k\right)\right)^{-1} \hat{q}_{\zeta}\right| & \leqq C s^{\alpha}\|\hat{q}\|_{\alpha_{N}}|\ln k||\xi|^{\alpha-\alpha^{\prime}}
\end{aligned}
$$

where the constants depend on $\varepsilon_{0}$ and $\alpha^{\prime}$, but not on $(\xi, \zeta, k)$.

To get the estimates in (1.9) for $\left(I+A_{1}\left(\hat{q}_{1}, k\right)\right)^{-1} g_{1}$ and $P\left(I+A_{1}\left(\hat{q}_{1}, k\right)\right)^{-1} g_{1}$, and thus for $h_{1}$ and $P h_{1}$, we need to repeat the arguments in [ER] deriving the "extra" smoothness of $g_{1}$ in $\xi$. In this case the extra smoothness takes the form

$$
\left\|\Delta(s) \Lambda_{\zeta}^{N} g_{1}\right\|_{\alpha} \leqq C s^{\alpha}|\ln k| \text { and }\left\|\Delta(v) \Lambda_{\zeta}^{N} g_{1}\right\|_{\alpha} \leqq C|v|^{\alpha}|\ln k|
$$

where \|\|$_{\alpha}$ denotes the norm in $\xi$ and $C$ is uniform for $(\xi, \zeta) \in \mathbb{R}^{2} \times \mathbb{R}^{2}$ and $0<k<\rho_{0}$. The estimates (1.10) are derived in the same manner as the extra smoothness estimates in [ER], see [ER, (3.21) and (3.23)]. Moreover, given (1.10), one proceeds to derive (1.9) for $h_{1}$ and $P h_{1}$ as in the final portion of the proof of Theorem 3.2 in [ER]. Thus we have:

Theorem 1.5. For $\hat{q} \in \mathcal{O}_{0}$ the solution $h_{1}(\xi, \zeta, k)$ of $h_{1}+A_{1}(\hat{q}, k) h_{1}=-\hat{q}_{\zeta}$ satisfies

$$
\begin{aligned}
\left|\Lambda_{\zeta}^{N} \Delta(v) h_{1}\right| & \leqq C|v|^{\alpha}|\ln k| \\
\left|\Lambda_{\zeta}^{N} \Delta(s) h_{1}\right| & \leqq C s^{\alpha} \ln k \mid, \\
\left|\Lambda_{\zeta}^{N} \Delta(v) P h_{1}\right| & \leqq C|v|^{\alpha}\left(1+|\xi|^{\alpha-\alpha^{\prime}}|\ln k|\right), \\
\left|\Lambda_{\zeta}^{N} \Delta(s) P h_{1}\right| & \leqq C s^{\alpha}|\xi|^{\alpha-\alpha^{\prime}}|\ln k|
\end{aligned}
$$

for $0<k<\rho_{0}<1,0<\alpha^{\prime}<\alpha$. The constants here depend on the choice of $\rho_{0}$ and $\alpha^{\prime}$ but are independent of $(\xi, \zeta, k)$.

\section{The Generic Form of the Backscattering Amplitude near Zero Energy}

As in $[E R]$ we define

$$
[A(\hat{q}, k) f](\xi)=(2 \pi)^{-2} \int_{\mathbb{R}^{2}} \frac{\hat{q}(\xi-\eta) f(\eta)}{|\eta|^{2}-(k+i 0)^{2}} d \eta .
$$

Then (I.1) can be written

$$
-\hat{q}(\xi-\zeta)=h(\xi, \zeta, k)+\left[A_{1}(\hat{q}, k) h\right](\xi)+\chi_{0}(k)[A(\hat{q}, k) \chi](\xi) h(0, \zeta, k),
$$

and, applying $\left(I+A_{1}\right)^{-1}$ to (2.1), it follows from (1.3) that for $k<\rho_{0}$,

$$
h(\xi, \zeta, k)-\left[A\left(h_{1}, k\right) \chi\right](\xi) h(0, \zeta, k)=h_{1}(\xi, \zeta, k),
$$

where

$$
\left[A\left(h_{1}, k\right) \chi\right](\xi)=(2 \pi)^{-2} \int_{\mathbb{R}^{2}} \frac{h_{1}(\xi, \eta, k) \chi(\eta)}{|\eta|^{2}-(k+i 0)^{2}} d \eta .
$$

Setting $\xi=0$ in $(2.2)$ and assuming $\left[A\left(h_{1}, k\right) \chi\right](0) \neq 1$ for $k<\rho_{0}$, we have

$$
h(0, \zeta, k)=\frac{h_{1}(0, \zeta, k)}{1-\left[A\left(h_{1}, k\right) \chi\right](0)},
$$


and from $(2.2)$

$$
\begin{aligned}
h(\xi, \zeta, k) & =h_{1}(\xi, \zeta, k)+\frac{\left[A\left(h_{1}, k\right) \chi\right](\xi) h_{1}(0, \zeta, k)}{1-\left[A\left(h_{1}, k\right) \chi\right](0)} \\
& =P h_{1}(\xi, \zeta, k)+\frac{h_{1}(0, \zeta, k) \chi(\xi)}{1-\left[A\left(h_{1}, k\right) \chi\right](0)}+\frac{h_{1}(0, \zeta, k)\left[P A\left(h_{1}, k\right) \chi\right](\xi)}{1-\left[A\left(h_{1}, k\right) \chi\right](0)} .
\end{aligned}
$$

Formula (2.4) will be used to analyze the singularities of $h(\xi,-\xi,|\xi|)$ for $|\xi|<\rho_{0}$. Note that the third and fourth estimates from Theorem 1.5 show that $P h_{1}(\xi,-\xi,|\xi|) \in H_{\alpha, N}$. The next step in the analysis is the study of $\left[A\left(h_{1}, k\right) \chi\right](0)$.

Defining

$$
a_{1}(k)=(2 \pi)^{-2} \int_{\mathbb{R}^{2}} \frac{\left(h_{1}(0, \eta, k)-h_{1}(0,0, k)\right) \chi(\eta)}{|\eta|^{2}-(k+i 0)^{2}} d \eta
$$

we have

$$
\left[A\left(h_{1}, k\right) \chi\right](0)=a_{1}(k)+\frac{h_{1}(0,0, k)}{(2 \pi)^{2}} \int_{\mathbb{R}^{2}} \frac{\chi(\eta)}{|\eta|^{2}-(k+i 0)^{2}} d \eta .
$$

Lemma 2.1. For $\hat{q} \in \mathcal{O}_{0}$ we have for $0<k<\rho_{0}<1$,

$$
\left|a_{1}(k)\right| \leqq C
$$

and

$$
\left|\Delta(s) a_{1}(k)\right| \leqq C s^{\alpha}|\ln k| .
$$

Proof. The estimate (2.6) is immediate from (2.5) and the estimate $\left\|h_{1}(0, \cdot k)\right\|_{\alpha^{\prime}} \leqq C$, $0<\alpha^{\prime}<\alpha$.

The proof (2.7) follows the proof of Theorem 1.5. In particular, if one substitutes the Neumann series for $\left(I+A_{1}\left(\hat{q}_{1}, k\right)\right)^{-1} \hat{q}_{\zeta}$ for $h_{1}$ in $(2.5)$ the general term is

$$
\begin{aligned}
I_{n}(k) & =\frac{(-1)^{n}}{(2 \pi)^{2}} \int_{\mathbb{R}^{2}} \frac{\left(\left[\left(A_{1}\left(\hat{q}_{1}, k\right)\right)^{n} \hat{q}_{\eta}\right](0)-\left[\left(A_{1}\left(\hat{q}_{1}, k\right)\right)^{n} \hat{q}\right](0)\right) \chi(\eta)}{|\eta|^{2}-(k+i 0)^{2}} d \eta \\
& =\frac{(-1)^{n}}{(2 \pi)^{2}} \int_{\mathbb{R}^{2}} \frac{\left[P_{1} A_{1}^{t}(\hat{q}, k)\left(A_{1}^{t}\left(\hat{q}_{1}, k\right)\right)^{n-1} f\right](\eta) \chi(\eta)}{|\eta|^{2}-(k+i 0)^{2}} d \eta,
\end{aligned}
$$

where $P_{1} g(\xi)=g(\xi)-g(0) \chi_{1}(\xi)$ with $\chi_{1} \in C_{0}^{\infty}, \chi_{1} \equiv 1$ on support $\chi$, and $f(\eta)=\hat{q}(-\eta)$. Thus, using Theorems 1.1 and 1.4 , one estimates $\Delta(s) I_{n}(k)$ as in (1.5), and completes the proof as in the proof of Theorem 1.5.

The estimate (2.7) does not imply that $a_{1}(|\xi|)$ is $C^{\alpha}$ at $\xi=0$. However, $\left(a_{1}(|\xi|)-a_{1}(0)\right)(\ln |\xi|)^{-1}$ is $C^{\alpha}$, as the following lemma shows.

Lemma 2.2. Assume that $a(\xi)$ is a continuous function on $|\xi| \leqq \delta<1$, satisfying

$$
|\Delta(\mu) a(\xi)| \leqq C|\mu|^{\alpha}(|\ln | \xi+\mu||+|\ln | \xi||) .
$$

Then $(a(\xi)-a(0))(\ln |\xi|)^{-1} \in C^{\alpha}(|\xi| \leqq \delta)$. In particular if $a(\xi) \in C^{\alpha}$, then $(a(\xi)-$ $a(0))(\ln |\xi|)^{-1} \in C^{\alpha}$.

Proof. We have for $|\xi| \leqq \delta$, and $n=0,1,2, \ldots$,

$$
\left|a\left(2^{-n} \xi\right)-a\left(2^{-n-1} \xi\right)\right| \leqq C 2^{-(n+1) \alpha}|\xi|^{\alpha}(2|\ln | \xi||+(2 n+1) \ln 2)
$$


and, since $|\xi| \leqq \delta$, summing on $n$ and changing the constant, this implies

$$
|a(\xi)-a(0)| \leqq C|\xi|^{\alpha}|\ln | \xi|| \text {. }
$$

Thus, letting $b(\xi)=(a(\xi)-a(0))(\ln |\xi|)^{-1}$, we have $|b(\xi)| \leqq C|\xi|^{\alpha}$. Since

$$
|\Delta(\mu) b(\xi)|=\left|\frac{a(\xi+\mu)-a(0)}{\ln |\xi+\mu|}-\frac{a(\xi)-a(0)}{\ln |\xi|}\right|,
$$

if $|\mu|<\frac{1}{2}|\xi|$, then

$$
\begin{aligned}
|\Delta(\mu) b(\xi)| & \leqq\left|\frac{a(\xi+\mu)-a(\xi)}{\ln |\xi|}\right|+|a(\xi+\mu)-a(0)|\left|\frac{\ln |\xi+\mu|-\ln |\xi|}{\ln |\xi| \ln |\xi+\mu|}\right| \\
& \leqq C|\mu|^{\alpha}+C \frac{|\xi+\mu|^{\alpha}}{|\ln | \xi||} \frac{|\mu|}{|\xi|} \leqq C|\mu|^{\alpha} .
\end{aligned}
$$

If $|\mu|>\frac{1}{2}|\xi|$, then $|\xi+\mu| \leqq 3|\mu|$, so that

$$
|\Delta(\mu) b(\xi)| \leqq|b(\xi+\mu)|+|b(\xi)| \leqq C|\xi+\mu|^{\alpha}+C|\xi|^{\alpha} \leqq C|\mu|^{\alpha} .
$$

The next lemma describes the remaining term in $\left[A\left(h_{1}, k\right) \chi\right](0)$.

Lemma 2.3. The function $\chi_{1}$, defined by

$$
(2 \pi)^{-2} \int_{\mathbb{R}^{2}} \frac{\chi(\eta)}{|\eta|^{2}-(k+i 0)^{2}} d n=-(2 \pi)^{-1} \ln k+\chi_{1}(k)
$$

belongs to $C^{\infty}[0, \infty)$.

Proof. We let $f(k)=(2 \pi)^{-1} \int_{S^{1}} \chi(k \omega) d \omega$. Then

$$
\begin{aligned}
& (2 \pi)^{-2} \int_{\mathbb{R}^{2}} \frac{\chi(\eta) d \eta}{|\eta|^{2}-(k+i 0)^{2}} \\
& =(4 \pi)^{-1} \int_{0}^{\infty} f(s) d\left(\ln \left(s^{2}-(k+i 0)^{2}\right)\right. \\
& =-(2 \pi)^{-1} \ln k+\frac{i}{4}-(4 \pi)^{-1} \int_{0}^{\infty} f^{\prime}(s) \ln \left(s^{2}-(k+i 0)^{2}\right) d s \\
& =-(2 \pi)^{-1} \ln k+\frac{i}{4}-(4 \pi)^{-1} \int_{0}^{\infty} f^{\prime}(s) \ln (k+s) d s \\
& \quad-(4 \pi)^{-1} \int_{0}^{\infty} f^{\prime}(s) \ln |k-s| d s+\frac{i}{4} \int_{0}^{k} f^{\prime}(s) d s \\
& =-(2 \pi)^{-1} \ln k+\frac{i}{4} f(k)-(4 \pi)^{-1} \int_{0}^{\infty} f^{\prime}(s) \ln \left|s^{2}-k^{2}\right| d s
\end{aligned}
$$

We are now ready to prove (I.2). Let $\mathcal{O}_{1}$ be the subset of $\mathcal{O}_{0}$ such that $h_{1}(0,0,0) \neq 0$ for $\hat{q} \in \mathcal{O}_{1}$. Note that Lemmas 2.1 and 2.3 imply $\left[A\left(h_{1}, k\right) \chi\right](0) \sim$ $-(2 \pi)^{-1} h_{1}(0,0,0) \ln k$ as $k \rightarrow 0$ so that $\left[A\left(h_{1}, k\right) \chi\right](0) \neq 1$ for $k<\varepsilon$ if $\varepsilon$ is sufficiently small when $\hat{q} \in \mathcal{O}_{1}$. We will discuss $\mathcal{O}_{0}$ and $\mathcal{O}_{1}$ further in Sect. 3.

Theorem 2.4. For $\hat{q} \in \mathcal{O}_{1}$ and $|\xi|$ sufficiently small, the scattering amplitude $b(\xi)=$ $h(\xi,-\xi,|\xi|)$ satisfies 


$$
b(\xi)=\frac{2 \pi}{\ln |\xi|+2 \pi \beta}+b_{1}(\xi),
$$

where $\beta=\left(1-a_{1}(0)-h_{1}(0,0,0) \chi_{1}(0)\right)\left(h_{1}(0,0,0)\right)^{-1}, b_{1}(0)=0$ and $b_{1} \in C^{\alpha}$.

Proof. Putting $\zeta=-\xi$ and $k=|\xi|$ in (2.4), we obtain

$$
\begin{aligned}
b(\xi)= & P h_{1}(\xi,-\xi,|\xi|)+\frac{h_{1}(0,0,0) \chi(\xi)}{1-\left[A\left(h_{1},|\xi|\right) \chi\right](0)} \\
& +\frac{\left(h_{1}(0,-\xi,|\xi|)-h_{1}(0,0,0)\right) \chi(\xi)}{1-\left[A\left(h_{1},|\xi|\right) \chi\right](0)}+\frac{h_{1}(0,-\xi,|\xi|)\left[P A\left(h_{1},|\xi|\right) \chi\right](\xi)}{1-\left[A\left(h_{1},|\xi|\right) \chi\right](0)} .
\end{aligned}
$$

As noted earlier, the estimates of Theorem 1.5 imply $P h_{1}(\xi,-\xi,|\xi|) \in C^{\alpha}$, and $P h_{1}(0,0,0)=0$ by the definition of $P$.

The singularity in $b(\xi)$ comes from the second term in the expansion (2.9). From (2.5) and the definition of $\chi_{1}$ one has

$$
\begin{gathered}
h_{1}(0,0,0)\left(1-\left[A\left(h_{1},|\xi| \chi\right](0)\right)^{-1}=\frac{2 \pi}{\ln |\xi|+2 \pi \beta}-\beta_{1}(\xi),\right. \\
\beta_{1}(\xi)=\frac{2 \pi}{\ln |\xi|+2 \pi \beta}-\frac{2 \pi h_{1}(0,0,0)}{h_{1}(0,0,|\xi|) \ln |\xi|+2 \pi\left(1-a_{1}(|\xi|)-h_{1}(0,0,|\xi|) \chi_{1}(|\xi|)\right)} .
\end{gathered}
$$

Note that the functions satisfying the hypotheses of Lemma 2.2 form an algebra which is closed under quotients as well when the denominators do not vanish. Since Theorem 1.5 and Lemma 2.1 show that $h_{1}(0,0,|\xi|)$ and $a_{1}(|\xi|)$ satisfy the hypotheses of Lemma 2.1, it follows that

$$
\beta_{1}=\frac{g_{1}+g_{2} \ln |\xi|}{f_{1}+f_{2} \ln |\xi|+(\ln |\xi|)^{2}},
$$

where the $f$ 's and $g$ 's satisfy the hypotheses of Lemma 2.2. Moreover, $g_{1}(0)=$ $g_{2}(0)=0$. Hence

$$
\beta_{1} \ln |\xi|=g_{2}+\frac{g_{1}-g_{2} f_{2}}{\ln |\xi|}+\frac{g_{3}}{f_{1}+f_{2} \ln |\xi|+(\ln |\xi|)^{2}},
$$

where $g_{3}$ has the properties of $g_{1}$ and $g_{2}$. By Lemma 2.2 it will follow that $\beta_{1} \in C^{\alpha}$, if we can show that $\left(f_{1}+f_{2} \ln |\xi|+(\ln |\xi|)^{2}\right)^{-1} g_{3}$ satisfies the hypotheses of Lemma 2.2. We have

$$
\begin{aligned}
& \left|\Delta(\mu)\left(\frac{g_{3}}{f_{1}+f_{2} \ln |\xi|+(\ln |\xi|)^{2}}\right)\right| \\
& \quad \leqq C \frac{\left|g_{3}(\xi)\right|}{|\ln | \xi|\ln | \xi+\mu||^{2}}\left|\Delta(\mu)\left(f_{1}+f_{2} \ln |\xi|+(\ln |\xi|)^{2}\right)\right|+C\left|\Delta(\mu) g_{3}\right| .
\end{aligned}
$$

The initial portion of the proof of Lemma 2.2 shows that $\left|g_{3}(\xi)\right| \leqq C|\xi|^{\alpha}|\ln | \xi \mid$. Since $|\Delta(\mu) \ln | \xi|| \leqq C|\mu||\xi|^{-1}$ when $|\mu|<\frac{1}{2}|\xi|$, one sees that

$$
\left|\Delta(\mu)\left(\left(f_{1}+f_{2} \ln |\xi|+(\ln |\xi|)^{2}\right)^{-1} g_{3}\right)\right| \leqq C|\mu|^{\alpha}(|\ln | \xi||+|\ln | \xi+\mu||)
$$


when $|\mu|<\frac{1}{2}|\xi|$. When $|\mu|>\frac{1}{2}|\xi|$, we simply use

$$
\left|\Delta(\mu)\left(f_{1}+f_{2} \ln \xi \mid+(\ln |\xi|)^{2}\right)\right| \leqq C\left(|\ln | \xi||^{2}+\left.\ln |\xi+\mu|\right|^{2}\right),
$$

and $\left|g_{3}(\xi)\right| \leqq C|\mu|^{\alpha}|\ln | \xi||$ to get the same estimate. Thus $\beta_{1} \in C^{\alpha}$.

Next we observe that

$$
\frac{\left(h_{1}(0,-\xi,|\xi|)-h_{1}(0,0,0)\right) \chi(\xi)}{1-\left[A\left(h_{1},|\xi|\right) \chi\right](0)}=\frac{g}{f+\ln |\xi|}=\frac{g \ln |\xi|}{f \ln |\xi|+(\ln |\xi|)^{2}},
$$

where $g$ and $f$ are as in (2.10). Thus the argument just given for $\beta_{1}$ shows this term is $C^{\alpha}$ as well.

Finally we have

$$
\begin{aligned}
& \frac{h_{1}(0,-\xi,|\xi|)\left[P A\left(h_{1},|\xi|\right) \chi\right](\xi)}{1-\left[A\left(h_{1},|\xi|\right) \chi\right](0)} \\
& \quad=\frac{h_{1}(0,-\xi,|\xi|)\left(a_{1}(\xi,|\xi|)-a_{1}(0,|\xi|) \chi(\xi)+P h_{1}(\xi, 0,|\xi|)\left(\frac{-1}{2 \pi} \ln |\xi|+\chi_{1}(|\xi|)\right)\right)}{1-\left[A\left(h_{1},|\xi|\right) \chi\right](0)},
\end{aligned}
$$

where

$$
a_{1}(\xi, k)=(2 \pi)^{-2} \int_{\mathbb{R}^{2}} \frac{h_{1}(\xi, \zeta, k)-h_{1}(\xi, 0, k)}{|\zeta|^{2}-(k+i 0)^{2}} \chi(\zeta) d \zeta .
$$

Note that $a_{1}(0, k)=a_{1}(k)$, the function introduced in $(2.5)$.

Theorem 1.5 implies that $P h_{1}(\xi, 0,|\xi|) \in C^{\alpha}$, and, since $P h_{1}(0,0,0)=0$, one checks easily that $P h_{1}(\xi, 0,|\xi|) \ln |\xi|$ satisfies the hypotheses of Lemma 2.2. Hence the right-hand side of (2.11) has exactly the form of the right-hand side of (2.10) (with $f_{1}$ and $g_{1}$ identically zero), provided that we can show $a_{1}(\xi,|\xi|)$ satisfies the hypotheses of Lemma 2.2 .

We claim that $a_{1}(\xi, k)$ satisfies the usual weighted estimates,

$$
\begin{aligned}
& \text { a) }\left|\Delta(\mu) a_{1}(\xi, k)\right| \leqq C|\mu|^{\alpha}|\ln k| \text {, and } \\
& \text { b) }\left|\Delta(s) a_{1}(\xi, k)\right| \leqq C s^{\alpha}|\ln k| \text {, }
\end{aligned}
$$

which imply $\left|\Delta(\mu) a_{1}(\xi,|\xi|)\right| \leqq C|\mu|^{\alpha}(|\ln | \xi||+|\ln | \xi+\mu||)$. To get b) we must again use the decomposition of $h_{1}$ into a Neumann series plus a term with extra smoothness. This argument is essentially the same as the proof of Lemma 2.1. In particular when one substitutes the Neumann series for $\left(I+A_{1}\left(\hat{q}_{1}, k\right)\right)^{-1} \hat{q}_{\zeta}$ for $h_{1}$ in the definition of $a_{1}(\xi, k)$, the general term is

$$
\begin{gathered}
\frac{(-1)^{n}}{(2 \pi)^{2}} \int_{\mathbb{R}^{2}} \frac{\left(\left[\left(A_{1}\left(\hat{q}_{1}, k\right)^{n} \hat{q}_{\zeta}\right](\xi)-\left[\left(A_{1}\left(\hat{q}_{1}, k\right)\right)^{n} \hat{q}\right](\xi)\right) \chi(\zeta)\right.}{|\zeta|^{2}-(k+i 0)^{2}} d \zeta \\
=\frac{(-1)^{n}}{(2 \pi)^{2}} \int_{\mathbb{R}^{2}} \frac{\left[P_{1} A_{1}^{t}(\hat{q}, k)\left(A_{1}^{t}\left(\hat{q}_{1}, k\right)\right)^{n-1} f\right](\eta) \chi(\eta)}{|\eta|^{2}-(k+i 0)^{2}} d \eta,
\end{gathered}
$$

where $f(\eta, \xi)=\hat{q}(\xi-\eta)$ and $P_{1} g(\xi)=g(\xi)-g(0) \chi_{1}(\xi)$ as the proof of Lemma 2.1 .

To prove a) we note that by the definition of $h_{1}, a_{1}(\xi, k)=\left[\left(I+A_{1}(\hat{q}, k)\right)^{-1} g\right](\xi)$, 
where

$$
g(\eta)=-(2 \pi)^{-2} \int_{\mathbb{R}^{2}} \frac{\left(\hat{q}(\eta-\zeta)-\chi_{1}(\zeta) \hat{q}(\eta)\right) \chi(\zeta) d \zeta}{|\zeta|^{2}-(k+i 0)^{2}}
$$

Thus, by the reasoning used to prove that

$$
\left\|A_{1}^{t}(p, k) f\right\|_{\alpha, N} \leqq C\|p\|_{\alpha, N}\|f\|_{\alpha, N}|\ln k|
$$

we have $\|g\|_{\alpha, N} \leqq C|\ln k|$. Since $\left(I+A_{1}(\hat{q}, k)\right)^{-1}$ is bounded on $H_{\alpha, N}$ by the definition of $\mathcal{O}_{0}$, we conclude $a_{1}(\xi, k)$ satisfies a). Thus $a_{1}(\xi,|\xi|)$ satisfies the hypotheses of Theorem 2.2, and we may use the argument given for $\beta_{1}$ for the final term in (2.9) as well.

\section{Analysis of the Hypotheses of Theorem 2.4}

The sets $\mathcal{O}$ and $\mathcal{O}^{\prime}$, defined by

$$
\begin{aligned}
\mathcal{O}=\{ & \left\{\hat{q} \in H_{\alpha, N}: I+A_{1}(\hat{q}, k) \text { is invertible on } H_{\alpha, N} \text { for } 0 \leqq k \leqq \rho_{0}\right. \text { and } \\
& \left.I+A(\hat{q}, k) \text { is invertible on } H_{\alpha, N} \text { for } k \geqq \rho_{1} \text { for some } \rho_{1}<\rho_{0}\right\}
\end{aligned}
$$

and

$$
\mathcal{O}^{\prime}=\left\{\hat{q} \in \mathcal{O}: h_{1}(0,0,0)=-\left[\left(I+A_{1}(\hat{q}, 0)\right)^{-1} \hat{q}\right](0) \neq 0\right\}
$$

will be studied here. Note that in Sect. 1 we defined $\mathcal{O}_{0}$ as the set of $\hat{q}$ such that $I+\Lambda_{\zeta}^{N} A_{1}(\hat{q}, k) \Lambda_{\zeta}^{-N}$ was invertible on $C^{\alpha}$ with the norm of its inverse uniformly bounded for $(\zeta, k) \in \mathbb{R}^{2} \times\left[0, \rho_{0}\right]$. The set $\mathcal{O}$ is a subset of $\mathcal{O}_{0}$ and for $\hat{q} \in \mathcal{O}$ we also have $I+\Lambda_{\zeta}^{N} A(\hat{q}, k) \Lambda_{\zeta}^{-N}$ invertible with inverse uniformly bounded for $(\zeta, k) \in \mathbb{R}^{2} \times$ $\left[\rho_{1}, \infty\right]$ (cf. [ER], Lemma 3.3). Hence $\mathcal{O}^{\prime}$ is a subset of the set $\mathcal{O}_{1}$ introduced in Sect. 2.

The set $\mathcal{O}$ is open in $H_{\alpha, N}$ by the arguments used to prove the corresponding result in Sect. 3 of [ER]. Since whether or not $\hat{q}$ belongs to $\mathcal{O}$ depends on $\chi$ and $\rho_{0}$, we are really interested in the set of $\hat{q}$ which belong to $\mathcal{O}$ for some choice of $\chi$ and $\rho_{0}$. As a union of open sets, this set, $\tilde{\mathcal{O}}$, is open. The following proposition shows that $\widetilde{\mathcal{O}}$ contains a dense subset of the Fourier transforms of real-valued potentials.

Proposition 3.1. Let $q$ be a real-valued function in $C_{0}^{\infty}\left(\mathbb{R}^{2}\right)$, and assume that there is no nontrivial solution $g_{0} \in C_{0}^{\infty}\left(\mathbb{R}^{2}\right)$ to

$$
g_{0}(x)+\frac{q(x)}{2 \pi} \int_{\mathbb{R}^{2}} \ln |x-y| g_{0}(y) d y=0
$$

satisfying $\int_{\mathbb{R}^{2}} g_{0}(x) d x=0$. Then there are $\chi$ and $\rho_{0}$ such that $\hat{q} \in \mathcal{O}$.

Proof. If $I+A(\hat{q}, k)$ fails to be invertible for some $k>0$, then there is a nontrivial solution to $-\Delta u+q u-k^{2} u=0$ satisfying the radiation condition. It is a classical result that such solutions do not exist for the $q$ considered here. Thus we only need to show there exist $\rho_{0}$ and $\chi$ such that $I+A_{1}(\hat{q}, k)$ is invertible for $0 \leqq k \leqq \rho_{0}$.

We begin by deriving some simple consequences of the hypothesis on $q$. The operator $q E_{0}$, where

$$
E_{0} f=(2 \pi)^{-1} \int_{\mathbb{R}^{2}} \ln |x-y| f(y) d y
$$


is compact on $L^{2}(|x|<R)$ for each $R$, and for $R$ greater than the support radius of $q$ the nullspace of $I+q E_{0}$ consists of $C_{0}^{\infty}$ functions with support contained in the support of $q$. Thus, if there is no solution to (3.3) with mean zero, there are two possibilities:

a) $I+q E_{0}$ is injective on $C_{0}^{\infty}\left(\mathbb{R}^{2}\right)$, or

b) the nullspace of $I+q E_{0}$ on $C_{0}^{\infty}\left(\mathbb{R}^{2}\right)$ is spanned by $g_{0}$ and $\int_{\mathbb{R}^{2}} g_{0} d x \neq 0$. In both cases the Fredholm alternative applies to $I+q E_{0}$ on $L^{2}(|x|<R)$. Hence, in case a) given $g \in C_{0}^{\infty}\left(\mathbb{R}^{2}\right)$, there is $h \in L^{2}(|x|<R)$ for $R$ sufficiently large such that $h+q E_{0} h=g$. It follows that $h$ belongs to $C_{0}^{\infty}\left(\mathbb{R}^{2}\right)$. Thus $I+q E_{0}$ maps $C_{0}^{\infty}\left(\mathbb{R}^{2}\right)$ onto $C_{0}^{\infty}\left(\mathbb{R}^{2}\right)$ in case a).

We can use (3.4) to define $E_{0}$ on the Schwartz space $\mathscr{S}\left(\mathbb{R}^{2}\right)$. Since

$$
\int_{\mathbb{R}^{2}} \ln |x-y| f(y) d y=\int_{\mathbb{R}^{2}} \ln |z| f(x-z) d z,
$$

we see that $q E_{0}$ maps $\mathscr{S}$ into $C_{0}^{\infty}\left(\mathbb{R}^{2}\right)$. In case a) we extend $\left(I+q E_{0}\right)^{-1}$ to $\mathscr{S}$ by defining

$$
\left(I+q E_{0}\right)^{-1} f=f-\left(I+q E_{0}\right)^{-1} q E_{0} f .
$$

Since $I+q E_{0}$ maps $\mathscr{S}$ into $\mathscr{S}$ and one may check easily that it is a two-sided inverse to $\left(I+q E_{0}\right)^{-1}$ as defined above, it follows that in case a) $\left(I+q E_{0}\right)$ is a linear isomorphism of $\mathscr{S}$.

We will now show that we can choose $\chi$ so that $I+A_{1}(\hat{q}, 0)$ is invertible. If $I+A_{1}(\hat{q}, 0)$ is not invertible, then by the compactness of $A_{1}(\hat{q}, 0)$ on $H_{\alpha, N}$ there is $\hat{h}_{0} \in H_{\alpha, N}$ in the nullspace of $I+A_{1}(\hat{q}, 0)$. Taking the inverse Fourier transform (denoted by "“", we have

$$
h_{0}+q E_{0}\left(h_{0}-\int_{\mathbb{R}^{2}} h_{0} d x \check{x}\right)=0
$$

which implies $h_{0}$ is in $C_{0}^{\infty}\left(\mathbb{R}^{2}\right)$. In case a) (3.5) implies

$$
\int_{\mathbb{R}^{2}} h_{0} d x=\int_{\mathbb{R}^{2}}\left(I+q E_{0}\right)^{-1} q E_{0} \check{\chi} d x \int_{\mathbb{R}^{2}} h_{0} d x .
$$

Since $\int_{\mathbb{R}} h_{0} d x \neq 0$, by hypothesis, we have

$$
1=\int_{\mathbb{R}^{2}}\left(I+q E_{0}\right)^{-1} q E_{0} \check{\chi} d x .
$$

If (3.6) holds for all admissible $\chi$, then, since any function in $C_{0}^{\infty}(\mathbb{R} \backslash 0)$ can be written as the difference of two admissible $\chi$ 's,

$$
0=\int_{\mathbb{R}^{2}}\left(1+q E_{0}\right)^{-1} q E_{0} \check{f} d x
$$

for all $f \in C_{0}^{\infty}\left(\mathbb{R}^{2}-\{0\}\right)$. Thus

$$
0=\int_{\mathbb{R}^{2}}\left(I+q E_{0}\right)^{-1} \check{f} d x
$$

for all $f \in C_{0}^{\infty}\left(\mathbb{R}^{2}-\{0\}\right)$. However, taking limits, one checks that (3.7) must hold for all $f \in \mathscr{S}_{0}=\{f \in \mathscr{S}: f(0)=0\}$, if it holds for all $f \in C_{0}^{\infty}\left(\mathbb{R}^{2}-\{0\}\right)$. Thus (3.8) holds for all $f \in \mathscr{S}_{0}$. Since $\mathscr{S}_{0}$ is codimension 1 in $\mathscr{S}$, if $\left(I+q E_{0}\right)^{-1}$ mapped $f \notin \mathscr{\mathscr { S }}_{0}$ into 
$\check{\mathscr{S}}_{0}$ it would necessarily map $\check{\mathscr{S}}$ into $\check{\mathscr{S}}_{0}$ contradicting the conclusion that $\left(I+q E_{0}\right)^{-1}$ is an isomorphism of $\mathscr{S}$. Thus if (3.6) holds for all admissible $\chi, 1+q E_{0}$ maps $\check{\mathscr{S}}_{0}$ onto $\check{\mathscr{S}}_{0}$ and, taking Fourier transforms we conclude

$$
0=\int_{\mathbb{R}^{2}} \frac{\hat{q}(-\eta) f(\eta)}{|\eta|^{2}} d \eta
$$

for all $f \in \mathscr{S}_{0}$. Thus $\hat{q} \equiv 0$, and $I+A_{1}(\hat{q}, 0)$ is trivially invertible.

In case b), since the nullspace of the adjoint of $I+q E_{0}$ in $L^{2}(|x|<R)$, when supp $q \subset\{|x| \subset R\}$, is spanned by $E_{0} g_{0}$ restricted to $|x|<R$, the Fredholm alternative applied to (3.5) implies

$$
0=\int_{\mathbb{R}^{2}}\left(E_{0} g_{0}\right) q E_{0} \check{\chi} d x .
$$

As in case a), if (3.10) holds for all admissible $\chi$, we have

$$
0=\int_{\mathbb{R}^{2}}\left(E_{0} g_{0}\right) q E_{0} \check{f} d x=\int_{\mathbb{R}^{2}} E_{0} g_{0} \check{f} d x
$$

for all $f \in \mathscr{S}_{0}$. Thus $E_{0} g_{0}$ must be constant. Since $\Delta\left(E_{0} g_{0}\right)=g_{0}$, and $g_{0} \notin 0$, this is a contradiction.

As we pointed out in the Introduction,

$$
E_{k}(x)=(2 \pi)^{-2} \int_{\mathbb{R}^{2}} \frac{e^{i x \cdot \eta}}{|\eta|^{2}-(k+i 0)^{2}} d \eta=\frac{i}{4}\left(J_{0}(k|x|)+i Y_{0}(k|x|) .\right.
$$

By standard results on Bessel functions, $E_{k}(x)=\left(\frac{-1}{2 \pi}+a(k|x|) k|x|\right) \ln k|x|+$ $b(k|x|)$, where $a(z)$ and $b(z)$ are entire functions and $\left|E_{k}(x)\right| \leqq C k^{-1 / 2}|x|^{-1 / 2}$ for $k|x|$ large. Thus $E_{k}(x)=\frac{-1}{2 \pi} \ln k+b(0)+E_{k}^{(1)}(x)$, where

$$
E_{k}^{(1)}(x)+\frac{1}{2 \pi} \ln |x| \underset{k \rightarrow 0}{\longrightarrow} 0
$$

uniformly on $|x| \leqq R$ for all $R$.

We now assume that $\chi$ has been chosen so that $I+A_{1}(\hat{q}, 0)$ is invertible. If $I+A_{1}(\hat{q}, k)$ has a nontrivial nullspace, then as before we have $g_{0} \in C_{0}^{\infty}$ with support contained in support $q$ satisfying

$$
g_{0}+q E_{k}\left(g_{0}-\int_{\mathbb{R}^{2}} g_{0} d x \check{\chi}\right)=0
$$

where $E_{k} f=\int E_{k}(x-y) f(y) d y$. Hence

$$
g_{0}+q E_{k}^{(1)}\left(g_{0}-\int_{\mathbb{R}} g_{0} d x \check{\chi}\right)=0 .
$$

However, choosing $R$ so that support $q$ is contained in $|x|<R$, as an operator on $L^{2}(|x|<R) q E_{k}^{(1)} \rightarrow q E_{0}$ in norm as $k \rightarrow 0$. Moreover,

$$
\left|E_{k}^{(1)}(x)+\frac{1}{2 \pi} \ln \right| x|| \leqq C k|x|(|\ln k| x||+1)
$$


for $k|x|<R$ and

$$
\left|E_{k}^{(1)}(x)+\frac{1}{2 \pi} \ln \right| x||<C+C|\ln | x||
$$

for $k|x|>R$. Since $\check{\chi} \in \mathscr{S}$, one sees easily that $q E_{k}^{(1)} \check{\chi}$ converges uniformly to $g E_{0} \check{\chi}$ as $k \rightarrow 0$. Thus, since invertibility of operators is preserved under small norm perturbations, it follows that there is $\rho_{0}>0$ such that for $0 \leqq k \leqq \rho_{0}$ there is no $g_{0} \in L_{2}(|x|<R)$ satisfying (3.11).

The set $\mathcal{O}^{\prime}$ is open in $H_{\alpha, N}$ : clearly $h_{1}(0,0,0)$ is a continuous function of $\hat{q}$ on the set of $\hat{q}$ such that $I+A(\hat{q}, 0)$ is invertible. To see that the union of the sets $\mathcal{O}^{\prime}$ over $\chi$ and $\rho_{0}$ contains dense subset of the Fourier transforms of real-valued potentials we will again consider potentials in $C_{0}^{\infty}\left(\mathbb{R}^{2}\right)$ with an additional constraint.

Proposition 3.2. Let $q$ be a real-valued function in $C_{0}^{\infty}\left(\mathbb{R}^{2}\right)$. Assume

a) there is no nontrivial solution $g_{0} \in C_{0}^{\infty}$ to

$$
g_{0}(x)+\frac{q(x)}{2 \pi} \int_{\mathbb{R}^{2}} \ln |x-y| g_{0}(y) d y=0, \quad \int_{\mathbb{R}^{2}} g_{0}(x) d x=0,
$$

b) there is no solution $h_{0} \in C_{0}^{\infty}\left(\mathbb{R}^{2}\right)$ to

$$
h_{0}(x)+\frac{q(x)}{2 \pi} \int_{\mathbb{R}^{2}} \ln |x-y| h_{0}(y) d y=q(x), \quad \int_{\mathbb{R}^{2}} h_{0}(x) d x=0 .
$$

Then there are $\chi$ and $\rho_{0}$ such that $\hat{q} \in \mathcal{O}^{\prime}$.

Proof. The requirement that $h_{1}(0,0,0) \neq 0$ is insured by b) for any choice of $\chi$ such that $I+A_{1}(\hat{q}, 0)$ is invertible: if $h_{1}(0,0,0)=0$, then the inverse Fourier transform of $h_{1}(\xi, 0,0)$ gives an $h_{0}(x)$ satisfying (3.13). Moreover, in the proof of Proposition 3.1 we showed that for any choice of $\chi$ such that $I+A_{1}(\hat{q}, 0)$ is invertible there will be a $\rho_{0}>0$ such that $I+A_{1}(\hat{q}, k)$ is invertible for $0 \leqq k \leqq \rho_{0}$. Thus, since hypothesis a) insures that there is a choice of $\chi$ such that $I+A_{1}(\hat{q}, 0)$ is invertible, we are done.

Remark 3.3. A slight variation of the proof of Proposition 3.1 shows that $\beta$ is not zero for real-valued $q \in C_{0}^{\infty}\left(\mathbb{R}^{2}\right)$ such that $\hat{q} \in \mathcal{O}^{\prime}$. One shows that, if $I+q E_{0}$ is invertible, there are cutoffs $\chi$ with arbitrarily small imaginary part such that $I+A_{1}(\hat{q}, 0)$ is invertible and $\check{\chi}$ is real-valued. Also, if $I+q E_{0}$ is not invertible but hypothesis a) of Proposition 3.2 holds, one can choose a cutoff $\chi$, such that $I+A_{1}(\hat{q}, 0)$ is invertible and both $\chi$ and $\check{\chi}$ are real-valued. Then, since $\check{\chi}$ is real-valued and, taking the inverse Fourier transform, $\breve{h}_{1}(x, 0,0)$ satisfies

$$
\check{h}_{1}+q E_{0}\left(\breve{h}_{1}-\int_{\mathbb{R}^{2}} \check{h}_{1} d x \check{\chi}\right)=-q,
$$

$\breve{h}_{1}(x, 0,0)$ is real-valued, and $h_{1}(0,0,0)$ is real. The constant $a_{1}(0)$ equals $g(0)$, where

$$
g=\left(I+A_{1}(\hat{q}, 0)\right)^{-1}\left((2 \pi)^{-2} \int_{\mathbb{R}^{2}} \frac{\hat{q}(\cdot)-\hat{q}(\cdot-\zeta)}{|\zeta|^{2}} \chi(\zeta) d \zeta\right)
$$


Taking inverse Fourier transforms

$$
\check{g}+q E_{0}\left(\check{g}-\int_{\mathbb{R}^{2}} \check{g} d x \check{\chi}\right)=q F,
$$

where, since $\check{\chi}$ is real-valued,

$$
F(x)=(2 \pi)^{-2} \int_{\mathbb{R}^{2}} \frac{1-e^{i \zeta \cdot x}}{|\zeta|^{2}} \chi(\zeta) d \xi
$$

is real-valued. Thus $\check{g}(x)$ is real-valued. By $(2.8) \chi_{1}(0)=\frac{i}{4}-(4 \pi)^{-1} \int_{0}^{\infty} f^{\prime}(s) \ln |s| d s=$ $\frac{i}{4}+c$, where $f(s)=(2 \pi)^{-1} \int_{S^{1}} \chi(s \omega) d \omega$. Thus

$$
\operatorname{Im} \beta=\left\{1-a_{1}(0)-h_{1}(0,0,0) \chi_{1}(0)\right\}=h_{1}(0,0,0)\left(\frac{1}{4}+\operatorname{Im} c\right) .
$$

Since we can choose $\operatorname{Im} \chi$ as small as we wish in $C_{0}^{\infty}\left(\mathbb{R}^{2}\right)$, we can make $\operatorname{Im} c$ as small as we wish. Hence, since $h_{1}(0,0,0) \neq 0, \operatorname{Im} \beta \neq 0$.

Remark 3.4. Note that the set of real potentials for which (3.12) has a nontrivial solution is of codimension 2 in the space of real-valued functions in $C_{0}^{\infty}\left(\mathbb{R}^{2}\right)$, while the set of real potentials such that (3.13) has a solution is of codimension 1 in the space of real-valued functions in $C_{0}^{\infty}\left(\mathbb{R}^{2}\right)$, and real codimension 2 in the space of complex-valued functions in $C_{0}^{\infty}\left(\mathbb{R}^{2}\right)$. This reasoning leads one to the conclusion that the set of real potentials satisfying the hypotheses of Proposition 3.1 is connected in real-valued $C_{0}^{\infty}\left(\mathbb{R}^{2}\right)$, and the set of real potentials satisfying the hypotheses of Proposition 3.2 is contained in a connected component of the union of the $\mathcal{O}^{\prime}$. A rigorous proof of this sort of result is given in Sect. 5 of [ER].

The representation $(2.4)$ for $h(\xi, \zeta, k)$ holds for $\hat{q} \in \mathcal{O}$ provided $\left[A\left(h_{1}, k\right) \chi\right](0) \neq 1$ for $0 \leqq k \leqq \rho_{0}$. We have

$$
\left[A\left[h_{1}, k\right) \chi\right](0)=a_{1}(k)+h_{1}(0,0, k)\left(\frac{-\ln k}{2 \pi}+\chi_{1}\right)
$$

so that there is clearly a $\rho^{\prime}$ such that

$$
\left[A\left(h_{1}, k\right) \chi\right](0) \neq 1 \text { for } 0 \leqq k \leqq \rho^{\prime} .
$$

Moreover, since $h_{1}(0,0, k)$ and $a_{1}(k)$, considered as elements of $C\left[0, \rho^{\prime}\right]$, depend continuously on $\hat{q}$, we see that for $\hat{q}_{0} \in \mathcal{O}^{\prime}$ there is a $\rho^{\prime}>0$ such that the representation (2.4) holds for all $\hat{q}$ in a neighborhood of $\hat{q}_{0}$ in $H_{\alpha, N}$ for $k \leqq \rho^{\prime}$. Thus for $|\xi|<\rho^{\prime}$ one has the representation of the backscattering given in Theorem 2.4 for all $\hat{q}$ in this neighborhood.

We can summarize the results of this section in the following way:

Theorem 3.5. For an open set $\mathscr{U}$ of $\hat{q}$ in $H_{\alpha, N}$ which has dense intersection with the set, $H_{\alpha, N}^{r}$, of Fourier transforms of real-valued functions, there are constants $r$ and $s$, $0<r<s$, locally independent of $\hat{q}$, such that the backscattering $b(\xi)$ has the representation (2.9) for $0<|\xi| \leqq s$ and hence

$$
b(\xi)=\frac{2 \pi}{\ln |\xi|+2 \pi \beta}+b_{1}(\xi), \quad b_{1} \in C^{\alpha}([0, s])
$$


with $b_{1}(0)=0$, and the representation for $|\xi| \geqq r$,

$$
b(\xi)=\left[(I+A(\hat{q},|\xi|))^{-1} \hat{q}_{-\xi}\right](\xi) .
$$

Remark 3.6. One can strengthen Proposition 3.2 by dropping the requirement that $q \in C_{0}^{\infty}\left(\mathbb{R}^{2}\right)$ and replacing (3.12) and (3.13) respectively by

a) there is no solution in $H_{\alpha, N} \cap\{\hat{f}(0)=0\}$ of

$$
\hat{g}(\xi)+(2 \pi)^{-2} \int_{\mathbb{R}^{2}} \frac{\hat{q}(\xi-\eta) \hat{g}(\eta)}{|\eta|^{2}} d \eta=0, \quad \text { and }
$$

b) there is no solution in $H_{\alpha, N} \cap\{\hat{f}(0)=0\}$ of

$$
\hat{h}_{0}(\xi)+(2 \pi)^{-2} \int_{\mathbb{R}^{2}} \frac{\hat{q}(\xi-\eta) \hat{h}_{0}(\eta)}{|\eta|^{2}} d \eta=-\hat{q}(\xi) .
$$

This does not change the conclusion of the proposition.

\section{The Frechet Derivative}

Theorem 3.5 only states that the backscattering mapping $S: \hat{q} \rightarrow \beta^{-1} \chi(\xi)+b_{0}(\xi)$ maps $\mathscr{U}$ into $H_{\alpha, N}$. However, the argument from Cauchy's formula used to prove Corollary 3.4 of [ER] applies here to show that $S$ is an analytic mapping of the open set $\mathscr{U}$ into $H_{\alpha, N}$. In particular, the Frechet derivative $d S(\hat{q})$ is a bounded operator on $H_{\alpha, N}$ depending continuously on $\hat{q}$. Our objective in this section is to show that, for $\hat{q} \in \mathscr{U}, d S(\hat{q})$ is a Fredholm operator of index zero. Since $C_{0}^{\infty}\left(\mathbb{R}^{2}\right)$ is dense in $H_{\alpha, N}$, it will suffice to prove this for $\hat{q} \in C_{0}^{\infty}\left(\mathbb{R}^{2}\right)$. Note that this implies $h_{1}(\xi, \zeta, k)$ is $C^{1-\varepsilon}$ in all variables for all $\varepsilon>0$. The arguments we present here will show that for $\hat{q} \in C_{0}^{\infty}, d S(\hat{q})=P+T$, where $P$ is invertible and $T P^{-1} T$ is compact on $H_{\alpha, N}$, provided $\alpha<\frac{1}{2}$. Hence $d S(\hat{q})$ is Fredholm of index zero when $\alpha<\frac{1}{2}$. The restriction on $\alpha$ is surely a result of our method of proof. $\left(T P^{-1}\right)^{n}$ is undoubtedly compact on $H_{\alpha, N}$ for $\alpha<1$ for $n$ sufficiently large, but we will not give a proof of that here.

It is also true for $k>0$ that $\hat{q} \rightarrow h(\cdot, \cdot, k)$ is a differentiable mapping from $\mathscr{U}$ to the space of functions satisfying

$$
\sup _{\zeta, \zeta}\left(\left\|\Lambda_{\zeta}^{N}(\cdot) f(\cdot, \zeta)\right\|_{\alpha}+\left\|\Lambda_{\zeta}^{N}(\cdot) f(\xi, \cdot)\right\|_{\alpha}\right)<\infty .
$$

We can use this to derive formulas for $d S(\hat{q})$ which will be needed later. Setting $\hat{q}(s)=\hat{q}+s v, v \in H_{\alpha, N}$, and, letting $\dot{f}$ denote $\frac{d f}{d s}(0)$, we have

$$
\dot{h}_{1}+A_{1}(\hat{q}, k) \dot{h}_{1}=-\dot{\hat{q}}_{\zeta}-A_{1}(\dot{\hat{q}}, k) h_{1} .
$$

Then, since $\left(I+A_{1}\left(h_{1}, k\right)\right)\left(I+A_{1}(\hat{q}, k)\right)=I$, we have

$$
\dot{h}_{1}=-\left(I+A_{1}\left(h_{1}, k\right)\right) \dot{\hat{q}}_{\zeta}-\left(I+A_{1}\left(h_{1}, k\right)\right) A_{1}(\dot{\hat{q}}, k) h_{1} .
$$

By (2.4) for $|\xi|<\delta$ the function $b_{0}(\xi)$ defined in the Introduction is given by

$$
b_{0}(\xi)=h_{1}(\xi,-\xi,|\xi|)+\frac{\left[A\left(h_{1},|\xi|\right) \chi\right](\xi)}{1-\left[A\left(h_{1},|\xi|\right) \chi\right](0)} h_{1}(0,-\xi,|\xi|)-\frac{2 \pi}{\ln |\xi|+2 \pi \beta} .
$$


Therefore, setting $f(\xi, k)=\left[A\left(h_{1}, k\right) \chi\right](\xi)$, we have for $\xi \neq 0$,

$$
\begin{aligned}
\dot{b}_{0}(\xi)= & \dot{h}_{1}(\xi,-\xi,|\xi|)+\frac{f(\xi,|\xi|)}{1-f(0,|\xi|)} \dot{h}_{1}(0,-\xi,|\xi|)+h_{1}(0,-\xi,|\xi|) \\
& \cdot\left(\frac{\left.A\left(\dot{h}_{1},|\xi|\right) \chi\right](\xi)}{1-f(0,|\xi|)}+\frac{f(\xi,|\xi|)}{(1-f(0,|\xi|))^{2}}\left[A\left(\dot{h}_{1},|\xi|\right) \chi\right](0)\right)+\frac{(2 \pi)^{2}}{(\ln |\xi|+2 \pi \beta)^{2}} \dot{\beta} \\
= & \dot{h}_{1}(\xi,-\xi,|\xi|)+\frac{f(\xi,|\xi|)}{1-f(0,|\xi|)} \dot{h}_{1}(0, \xi,|\xi|)+(2 \pi)^{-2} \frac{h_{1}(0,-\xi,|\xi|)}{1-f(0,|\xi|)} \\
& \cdot \int_{\mathbb{R}^{2}}\left(\dot{h}_{1}(\xi, \eta,|\xi|)+\frac{f(\xi,|\xi|)}{1-f(0,|\xi|)} \dot{h}_{1}(0, \eta,|\xi|)\right) \frac{\chi(\eta)}{|\eta|^{2}-(|\xi|+i 0)^{2}} d \eta \\
& +\frac{(2 \pi)^{2}}{(\ln |\xi|+2 \pi / \beta)^{2}} \dot{\beta} .
\end{aligned}
$$

We exclude $\xi=0$ because several terms in (4.3) are undefined at $\xi=0$. However, combining (4.1) and (4.3) we necessarily get an expression for $[d S(\hat{q}) \dot{\hat{q}}](\xi)$ for $0<|\xi| \leqq \delta$. Since $d S(\hat{q})$ restricted to $|\xi|<\delta$ is a bounded mapping from $H_{\alpha, N}$ to $C^{\alpha}(|\xi| \leqq \delta)$, the expression for $\dot{b}_{0}(\xi)$ derived from (4.1) and (4.3) must extend to a $C^{\alpha}$ function on $|\xi|<\delta$, though this involves cancellations which are not yet obvious.

In the decomposition $d S(\hat{q})=P+T$, the operator $P$ will be given by $[P \dot{q}](\xi)=$ $-\dot{\hat{q}}(2 \xi)$. In analyzing $T$ we will make much use of the fact that operator norm limits of compact operators are compact. In particular, modulo operators with one-dimensional range, we can decompose $T: H_{\alpha, N} \rightarrow C^{\alpha}(|\xi| \leqq \delta)$ as a sum of operators $T_{i}$ with the property that $\left[T_{i} f\right](0)=0$. This is completed in (4.11). Most of the $T_{i}$ 's will have the additional property that the norm of $T_{i}$ as an operator from $H_{\alpha, N}$ to $C^{\alpha}(|\xi| \leqq \delta)$ goes to zero as $\delta$ goes zero. Denote the sum over these $T_{i}$ 's as $\sum^{\prime \prime} T_{i}$ and the sum over the rest as $\sum^{\prime \prime} T_{i}$. Our strategy will be as follows. For any $\rho \in C_{0}^{\infty}$ such that $\rho=1$ in a neighborhood of $\xi=0$ we will show (Prop. 4.1) that $(1-\rho) T P^{-1}(1-\rho) T$ is compact. From the preceeding it is clear that $\rho \sum^{\prime} T_{i}$ can be given arbitrarily small norm by taking the support of $\rho$ sufficiently close to zero. Finally we will show (Prop. 4.2) $\rho\left(\sum^{\prime \prime} T_{i}\right) P^{-1} \rho\left(\sum^{\prime \prime} T_{i}\right)$ is a sum of compact operators and operators whose norm tends to zero as the support of $\rho$ shrinks to $\xi=0$. Since the operators $(1-\rho) T P^{-1} \rho\left(\sum^{\prime \prime} T_{i}\right)$ and $\rho\left(\sum^{\prime \prime} T_{i}\right) P^{-1}(1-\rho) T$ are also compact (Prop. 4.3), it will follow that $T P^{-1} T$ is the norm limit of compact operators.

We will begin by simply going through the terms in (4.3), and regrouping them to form $T_{i}$ 's. In view of the structure of the terms we need to begin with

$$
\begin{aligned}
& \dot{h}_{1}(\xi, \zeta, k)+\frac{f(\xi, k)}{1-f(0, k)} \dot{h}_{1}(0, \zeta, k) \\
& \quad=\dot{h}_{1}(\xi, \zeta, k)-\dot{h}_{1}(0, \zeta, k)+\frac{1}{1-f(0, k)} \dot{h}_{1}(0, \zeta, k)+\frac{f(\xi, k)-f(0, k)}{1-f(0, k)} \dot{h}_{1}(0, \zeta, k) \\
& \quad=-\dot{\hat{q}}(\xi-\zeta)+\dot{\hat{q}}(-\zeta)+\frac{1}{1-f(0, k)} \dot{h}_{1}(0, \zeta, k)+\left[T_{0} \dot{\hat{q}}\right](\xi, \zeta, k) .
\end{aligned}
$$


We note that all contributions to $\left[T_{0} \dot{\hat{q}}\right](\xi, \zeta, k)$ except $-\left[A_{1}(\dot{\hat{q}}, k) h_{1}\right](\xi, \zeta, k)+$ $\left.\left[A_{1}(\dot{\hat{q}}, k) h_{1}\right]\right)(0, \zeta, k)$ will contribute operators of arbitrarily small norm to $d S(\hat{q})$, since they all contain factors of $h_{1}(\xi, \eta, k)-h_{1}(0, \eta, k)$. Expanding further

$$
\begin{aligned}
\frac{1}{1-f(0,|\xi|)} \dot{h}_{1}(0, \zeta,|\xi|)= & \frac{1}{1-f(0,|\xi|)}(-\dot{q}(-\zeta)+f(0,|\xi|)(\dot{q}(-\zeta)-\dot{q}(0)) \\
& -\left[B_{1} \dot{\hat{q}}\right](0, \zeta,|\xi|)-\left[B_{2} \dot{\hat{q}}\right](\zeta,|\xi|),
\end{aligned}
$$

where

$$
B_{1} \dot{\hat{q}}=\left(I+A_{1}\left(h_{1},|\xi|\right)\right) A_{1}(\dot{\hat{q}},|\xi|) h_{1}
$$

and

$$
\left[B_{2} \dot{\hat{q}}\right](\zeta,|\xi|)=(2 \pi)^{-2} \int_{\mathbb{R}^{2}} \frac{h_{1}(0, \eta,|\xi|)(\dot{\hat{q}}(\eta-\zeta)-\dot{\hat{q}}(0) \chi(\eta))}{|\eta|^{2}-(|\xi|+i 0)^{2}} d \eta .
$$

We now have in place of (4.4):

$$
\begin{aligned}
& \dot{h}_{1}(\xi, \zeta,|\xi|)+\frac{f(\xi,|\xi|)}{1-f(0,|\xi|)} \dot{h}_{1}(0, \zeta,|\xi|) \\
& =-\dot{\hat{q}}(\xi-\zeta)+\frac{-1}{1-f(0,|\xi|)}\left(f(0,|\xi|) \dot{q}(0)+\left[B_{1} \hat{q}\right](0, \zeta,|\xi|)\right. \\
& \left.\quad+\left[B_{2} \dot{\hat{q}}\right](\zeta,|\xi|)\right)+\left[T_{0} \dot{\hat{q}}\right](\xi, \zeta,|\xi|) .
\end{aligned}
$$

At this point it may be helpful to consider what must happen when we substitute (4.6) into (4.3). The operator $T_{0}$ is quite well-behaved, and, since $\left[T_{0} \dot{\hat{q}}\right](0, \zeta,|\xi|)=0$, we will never need to expand it further. The operator $B_{1}$ is also well-behaved, but we will need to expand $\left[B_{1} \dot{\hat{q}}\right](0,-\xi,|\xi|)$ as

$$
\left(\left[B_{1} \dot{\hat{q}}\right](0,-\xi,|\xi|)-\left[B_{1} \dot{\hat{q}}\right](0,0,0)\right)+\left[B_{1} \dot{\hat{q}}\right](0,0,0)
$$

to split $(1-f(0,|\xi|))^{-1}\left[B_{1} \dot{\hat{q}}\right](0,-\xi,|\xi|)$ into the sum of an operator $T_{1}$ and a rank one operator. This is also true of $\left(1-f(0,|\xi|)^{-1}\left[B_{2} \dot{\hat{q}}\right](-\xi,|\xi|)\right.$. In the integral term in (4.3) there are further complications. We will expand $\left[B_{1} \dot{\hat{q}}\right](0, \eta,|\xi|)$ as $\left(\left[B_{1} \dot{\hat{q}}\right](0, \eta,|\xi|)-\left[B_{1} \dot{\hat{q}}\right](0,0,|\xi|)-\left[B_{1} \dot{\hat{q}}\right](0, \eta, 0)+\left[B_{1} \dot{\hat{q}}\right](0,0,0)\right)+\left(\left[B_{1} \dot{\hat{q}}\right](0,0,|\xi|)\right.$

$$
\left.-\left[B_{1} \dot{\hat{q}}\right](0,0,0)\right)+\left(\left[B_{1} \dot{\hat{q}}\right](0, \eta, 0)-\left[B_{1} \dot{\hat{q}}\right](0,0,0)\right)+\left[B_{1} \dot{\hat{q}}\right](0,0,0) \text {. }
$$

However, $\left[B_{2} \hat{q}\right](\eta, 0)$ is undefined, and we cannot use this expansion for $\left[B_{2} \hat{q}\right](\eta,|\xi|)$. We will need to expand the part of the integral term in (4.2) involving $\left[B_{2} \hat{q}\right](\eta,|\xi|)$ as a further sum of integrals.

Setting

$$
\left[B_{3} \dot{\hat{q}}\right](\xi,|\xi|)=(2 \pi)^{-2} \int_{\mathbb{R}^{2}} \frac{(\dot{\hat{q}}(\xi-\eta)-\dot{\hat{q}}(0)) \chi(\eta)}{|\eta|^{2}-(|\xi|+i 0)^{2}} d \eta
$$

and

$$
m(|\xi|)=(2 \pi)^{-2} \int_{\mathbb{R}^{2}} \frac{\chi\left(\left.\eta\right|^{2}-(|\xi|+i 0)^{2}\right.}{\mid \eta,}
$$


(4.3) can now be written

$$
\begin{aligned}
\dot{b}_{0}(\xi)= & \left.-\dot{\hat{q}}(2 \xi)+\frac{-1}{1-f(0,|\xi|)}\left(f(0,|\xi|) \dot{\hat{q}}(0)+\left[B_{1} \dot{\hat{q}}\right]\right](0,-\xi,|\xi|)+\left[B_{2} \dot{\hat{q}}\right](-\xi,|\xi|)\right) \\
& +\left[T_{0} \dot{\hat{q}}\right](\xi,-\xi,|\xi|)-\frac{h_{1}(0,-\xi,|\xi|)}{1-f(0,|\xi|)}\left(\left[B_{3} \dot{\hat{q}}\right](\xi,|\xi|)+\frac{m(|\xi|) \dot{\hat{q}}(0)}{1-f(0,|\xi|)}\right. \\
& +\frac{(2 \pi)^{-2}}{1-f(0,|\xi|)}\left(\int_{\mathbb{R}^{2}} \frac{\left[B_{1} \dot{\hat{q}}\right](0, \eta,|\xi|) \chi(\eta)}{|\eta|^{2}-(|\xi|+i 0)^{2}} d \eta+\int_{\mathbb{R}^{2}} \frac{\left[B_{2} \dot{\hat{q}}\right](\eta,|\xi|) \chi(\eta)}{|\eta|^{2}-(|\xi|+i 0)^{2}} d \eta\right) \\
& \left.-(2 \pi)^{-2} \int_{\mathbb{R}^{2}} \frac{\left[T_{0} \dot{\hat{q}}\right](\xi, \eta,|\xi|) \chi(\eta)}{|\eta|^{2}-(|\xi|+i 0)^{2}} d \eta\right)+\frac{(2 \pi)^{2}}{(\ln |\xi|+2 \pi \beta)^{2}} \dot{\beta} .
\end{aligned}
$$

Only three of the terms in (4.7), $-\dot{\hat{q}}(2 \xi),\left[T_{0} \dot{\hat{q}}\right](\xi,-\xi,|\xi|)$ and the integral involving $T_{0}$, are operators mapping $\hat{q} \in H_{\alpha, N}$ boundedly into $C^{\alpha}(|\xi| \leqq \delta)$. However, we can show each terms is the sum of a bounded operator from $H_{\alpha, N}$ to $C^{\alpha}(|\xi| \leqq \delta)$ and one or more rank one operators. To state this more precisely we introduce

$\left[T_{1} \dot{\hat{q}}\right](\xi, k)=-(1-f(0, k))^{-1}\left(\left[B_{1} \dot{\hat{q}}\right](0,-\xi, k)-\left[B_{1} \dot{\hat{q}}\right](0,0,0)\right)$,

$\left[T_{2} \dot{\hat{q}}\right](\xi, k)=-(1-f(0, k))^{-1}\left(\left[B_{2} \dot{\hat{q}}\right](-\xi, k)-\left[B_{2} \dot{\hat{q}}\right](0,0)\right)$,

$\left[T_{3} \dot{\hat{q}}\right](\xi, k)=-(1-f(0, k))^{-1} h_{1}(0,-\xi, k)\left(\left[B_{3} \dot{\hat{q}}\right](\xi, k)-\left[B_{3} \dot{\hat{q}}\right](0,0)\right)$,

$\left[T_{4} \dot{\hat{q}}\right](\xi, k)=-(1-f(0, k))^{-2} h_{1}(0,-\xi, k)$

$$
\begin{aligned}
& \cdot\left((2 \pi)^{-2} \int_{\mathbb{R}^{2}} \frac{\left(\left[B_{2} \dot{\hat{q}}\right](\eta, k)-\left[B_{2} \dot{q}\right](0, k)\right) \chi(\eta)}{|\eta|^{2}-(k+i 0)^{2}} d \eta-f(0, k)\left[B_{3} \dot{\hat{q}}\right](0, k)\right. \\
& \left.-(2 \pi)^{-4} \int_{\mathbb{R}^{2}} \int_{\mathbb{R}^{2}} \frac{\chi(\eta) h_{1}(0, \zeta, 0)(\dot{\hat{q}}(\zeta-\eta)-\dot{\hat{q}}(\zeta)-(\dot{\hat{q}}(-\eta)-\dot{\hat{q}}(0)) \chi(\zeta))}{|\eta|^{2}|\zeta|^{2}} d \eta d \zeta\right),
\end{aligned}
$$

$\left[T_{5} \dot{\hat{q}}\right](\xi, k)=h_{1}(0,-\xi, k) m(k)(1-f(0, k))^{-1}\left[T_{2} \dot{\hat{q}}\right](0, k)$,

$\left[T_{6} \dot{\hat{q}}\right](\xi, k)=h_{1}(0,-\xi, k) f(0, k)(1-f(0, k))^{-1}\left[T_{3} \dot{\hat{q}}\right](0, k)$,

$\left[T_{7} \dot{\hat{q}}\right](\xi, k)=-(1-f(0, k))^{-2} h_{1}(0,-\xi, k)$

$$
\begin{aligned}
& \cdot\left((2 \pi)^{-2} \int_{\mathbb{R}^{2}} \frac{\left(\left[B_{1} \dot{\hat{q}}\right](0, \eta, k)-\left[B_{1} \dot{\hat{q}}\right](0,0, k)\right) \chi(\eta)}{|\eta|^{2}-(k+i 0)^{2}} d \eta\right. \\
& \left.-(2 \pi)^{-2} \int_{\mathbb{R}^{2}} \frac{\left(\left[B_{1} \dot{\hat{q}}\right](0, \eta, 0)-\left[B_{1} \dot{\hat{q}}\right](0,0,0)\right) \chi(\eta)}{|\eta|^{2}} d \eta\right),
\end{aligned}
$$

and

$$
\left[T_{8} \dot{\hat{q}}\right](\xi, k)=h_{1}(0,-\xi, k) m(k)(1-f(0, k))^{-1}\left[T_{1} \dot{\hat{q}}\right](0, k) .
$$

Setting

$$
\left[T_{9} \dot{\hat{q}}\right](\xi, k)=\frac{h_{1}(0,-\xi, k)}{(2 \pi)^{2}(1-f(0, k))} \int_{\mathbb{R}^{2}} \frac{\left[T_{0} \dot{\hat{q}}\right](\xi, \eta, k) \chi(\eta)}{|\eta|^{2}-(k+i 0)^{2}} d \eta
$$

we can write (4.7) as

$$
\dot{\hat{b}}_{0}=-\dot{\hat{q}}(2 \xi)+\frac{-1}{1-f(0,|\xi|)}\left(f(0,|\xi|) \dot{q}(0)+\left[B_{1} \dot{\hat{q}}\right](0,0,0)+\left[B_{2} \dot{\hat{q}}\right](0,0)\right)
$$




$$
\begin{aligned}
& -\frac{h_{1}(0,-\xi,|\xi|)}{\left(1-f(0,|\xi|)^{2}\right.}\left(\left[B_{3} \dot{\hat{q}}\right](0,0)+m(|\xi|) \dot{\hat{q}}(0)\right. \\
& +(2 \pi)^{-4} \int_{\mathbb{R}^{2}} \int_{\mathbb{R}^{2}} \frac{\chi(\eta) h_{1}(0, \zeta, 0)(\dot{\hat{q}}(\zeta-\eta)-\dot{\hat{q}}(\zeta)-(\dot{\hat{q}}(-\eta)-\dot{\hat{q}}(0)) \chi(\zeta))}{|\eta|^{2}|\zeta|^{2}} d \eta d \zeta \\
& +m(|\xi|)\left[B_{2} \dot{\hat{q}}\right](0,0)+(2 \pi)^{-2} \int_{\mathbb{R}^{2}} \frac{\left(\left[B_{1} \dot{\hat{q}}\right](0, \eta, 0)-\left[B_{1} \dot{\hat{q}}\right](0,0,0)\right) \chi(\eta)}{|\eta|^{2}} d \eta \\
& \left.+m(|\xi|)\left[B_{1} \dot{\hat{q}}\right](0,0,0)\right)+\frac{(2 \pi)^{2}}{(\ln |\xi|+2 \pi \beta)^{2}} \dot{\beta}+\sum_{i=0}^{9}\left[T_{i} \dot{\hat{q}}\right](\xi,|\xi|) .
\end{aligned}
$$

We need to identify the cancellations in (4.8). Some of these are consequences of the following simple observation:

$$
\begin{aligned}
f(0,|\xi|) & =(2 \pi)^{-2} \int_{\mathbb{R}^{2}} \frac{h_{1}(0, \eta,|\xi|) \chi(\eta)}{\left.|\eta|^{2}-(|\xi|)+i 0\right)^{2}} d \eta \\
& =h_{1}(0,0,0) m(|\xi|)+(2 \pi)^{-2} \int_{\mathbb{R}^{2}} \frac{\left(h_{1}(0, \eta,|\xi|)-h_{1}(0,0,0)\right) \chi(\eta)}{|\eta|^{2}-(|\xi|+i 0)^{2}} .
\end{aligned}
$$

Thus

$$
\frac{f(0,|\xi|)}{1-f(0,|\xi|)}=\frac{h_{1}(0,0,0) m(|\xi|)}{1-f(0,|\xi|)}+\frac{a_{1}(0)}{1-f(0,|\xi|)}+Q(\xi),
$$

where $Q(\xi)$ is a function whose norm goes to zero in $C^{\alpha}(|\xi| \leqq \delta)$ as $\delta \rightarrow 0$. Keeping in mind that $\left(h_{1}(0,-\xi,|\xi|)-h_{1}(0,0,0)\right)\left(1-f(0,|\xi|)^{-1} m(|\xi|)\right.$ is also a function whose norm goes to zero in $C^{\alpha}(|\xi| \leqq \delta)$ as $\delta \rightarrow 0$ and collecting terms of arbitrarily small norm as $T_{10} \dot{\hat{q}},(4.8)$ becomes

$$
\begin{aligned}
& \dot{b}_{0}(\xi)=-\dot{\hat{q}}(2 \xi)+\dot{\hat{q}}(0)-\frac{\dot{\hat{q}}(0)}{(1-f(0,|\xi|))^{2}}+\frac{a_{1}(0) \dot{\hat{q}}(0)}{(1-f(0,|\xi|))^{2}} \\
& -\frac{1}{(1-f(0,|\xi|))^{2}}\left(\left(1-a_{1}(0)\right)\left[B_{1} \dot{\hat{q}}\right](0,0,0)+\left(1-a_{1}(0)\right)\left[B_{2} \dot{\hat{q}}\right](0,0)\right. \\
& +h_{1}(0,0,0)(2 \pi)^{-4} \int_{\mathbb{R}^{2}} \int_{\mathbb{R}^{2}} \frac{\chi(\eta) h_{1}(0, \zeta, 0)(\dot{\hat{q}}(\zeta-\eta)-\dot{\hat{q}}(\zeta)-(\dot{\hat{q}}(-\eta)-\dot{\hat{q}}(0)) \chi(\zeta))}{|\eta|^{2}|\zeta|^{2}} d \eta d \zeta \\
& +h_{1}(0,0,0)(2 \pi)^{-2} \int_{\mathbb{R}^{2}}\left(\frac{\left[B_{1} \dot{\hat{q}}\right](0, \eta, 0)-\left[B_{1} \dot{\hat{q}}\right](0,0,0)}{|\eta|^{2}}\right) \chi(\eta) d \eta \\
& \left.+h_{1}(0,0,0)\left[B_{3} \dot{\hat{q}}\right](0,0)\right)+\frac{(2 \pi)^{2}}{(\ln |\xi|+2 \pi \beta)^{2}} \dot{\beta}+\sum_{i=0}^{10}\left[T_{i} \dot{\hat{q}}\right](\xi) \text {. }
\end{aligned}
$$

Next we recall that:

a)

$$
\beta=\frac{1-a_{1}(0)}{h_{1}(0,0,0)}+\chi_{1}(0)
$$


and hence

$$
\begin{aligned}
\dot{\beta} & =\frac{-\dot{a}_{1}(0)}{h_{1}(0,0,0)}-\frac{1-a_{1}(0)}{h_{1}^{2}(0,0,0)} \dot{h}_{1}(0,0,0) \\
& =\frac{-(2 \pi)^{-2}}{h_{1}(0,0,0)} \int_{\mathbb{R}^{2}} \frac{\left(\dot{h}_{1}(0, \eta, 0)-\dot{h}_{1}(0,0,0)\right) \chi(\eta)}{|\eta|^{2}} d \eta-\frac{1-a_{1}(0)}{h_{1}^{2}(0,0,0)} \dot{h}_{1}(0,0,0),
\end{aligned}
$$

and

b)

$$
\frac{h_{1}(0,0,0)}{1-f(0,|\xi|)}=\frac{2 \pi}{\ln |\xi|+2 \pi \beta}+\beta_{1}(\xi),
$$

where $\beta_{1}(0)=0$ and $\beta_{1} \in C^{\alpha^{\prime}}$ for all $\alpha^{\prime}<1$. Using (4.1) to evaluate $\dot{h}_{1}$, one sees from (4.10) that the coefficient of $(1-f(0,|\xi|))^{-2}$ in (4.9) is $-h_{1}^{2}(0,0,0) \dot{\beta}$, and, using b) one concludes that these terms cancel against $(2 \pi)^{2}(\ln |\xi|+2 \pi \beta)^{-2} \dot{\beta}$ modulo a final error term of small norm, $T_{11} \dot{\hat{q}}$. Thus we have

$$
\dot{b}_{0}(\xi)=-\dot{\hat{q}}(2 \xi)+\dot{\hat{q}}(0)+\sum_{i=0}^{11}\left[T_{i} \dot{\hat{q}}\right](\xi,|\xi|) .
$$

At this point we have twelve operators $T_{i}$ to analyze. Most of the $T_{i}$ have norms, as operators from $H_{\alpha, N}$ to $C^{\alpha}(|\xi| \leqq \delta$ ) which tend to zero as $\delta$ goes to zero, and are therefore part of $\sum^{\prime}$. To make this precise, we list the following observations.

i) The operators $T_{10}$ and $T_{11}$ clearly have norms tending to zero with $\delta$ and do not require further discussion.

ii) The operators $T_{2}, T_{3}, T_{5}$ and $T_{6}$ are of the form

$$
[T \hat{q}](\xi)=g(\xi)(1-f(0,|\xi|))^{-1}(J(\xi)-J(0)),
$$

where

$$
J(\xi)=\int_{\mathbb{R}^{2}} \frac{\tau_{1}(\xi, \eta) \dot{\hat{q}}(\xi-\eta)-\tau_{2}(\xi, \eta) \dot{\hat{q}}(0)}{|\eta|^{2}-(|\xi|+i 0)^{2}} d \eta .
$$

The coefficients $\tau_{i}, i=1,2$, are in $C^{\beta}$ for all $\beta<1$, and are of rapid decrease as $|\eta| \rightarrow \infty$. These properties follow from the assumption that $\hat{q} \in C_{0}^{\infty}\left(\mathbb{R}^{2}\right)$. We also have $\tau_{1}(0,0)=\tau_{2}(0,0)$. The function $g(\xi)$ is of the form

$$
g(\xi)=\frac{a}{1-f(0,|\xi|)}+w(\xi)
$$

where $a \in \mathbb{C}$ and $w \in C^{\beta}\left(\mathbb{R}^{2}\right)$ for all $\beta<1$.

To simplify these terms we expand

$$
\begin{aligned}
J(\xi)= & \tau_{1}(0,0) \int_{\mathbb{R}^{2}} \frac{\dot{\hat{q}}(\xi-\eta)-\dot{\hat{q}}(0) \chi(\eta)}{|\eta|^{2}-(|\xi|+i 0)^{2}} d \eta \\
& +\int_{\mathbb{R}^{2}} \frac{\left(\tau_{1}(0, \eta)-\tau_{1}(0,0)\right)(\dot{\hat{q}}(\xi-\eta)-\dot{q}(0) \chi(\eta))}{|\eta|^{2}-(|\xi|+i 0)^{2}} d \eta \\
& +\int_{\mathbb{R}^{2}} \frac{\left(\tau_{1}(\xi, \eta)-\tau_{1}(0, \eta)\right) \dot{\hat{q}}(\xi-\eta)-\left(\tau_{2}(\xi, \eta)-\tau_{1}(0, \eta)\right) \dot{q}(0)}{|\eta|^{2}-(|\xi|+i 0)^{2}} d \eta \\
= & J_{1}(\xi)+J_{2}(\xi)+J_{3}(\xi) .
\end{aligned}
$$

Theorems 1.1 and 1.4 imply directly that $J_{2}$ is bounded from $H_{\alpha, N}$ to $C^{\alpha}(|\xi| \leqq \delta)$. 
We expand $J_{3}$ as

$$
\begin{aligned}
J_{3}(\xi)= & \int_{\mathbb{R}^{2}} \frac{\left(\tau_{1}(\xi, \eta)-\tau_{1}(0, \eta)\right)(\dot{\hat{q}}(\xi-\eta)-\dot{q}(\xi))}{|\eta|^{2}-(|\xi|+i 0)^{2}} d \eta \\
& +\dot{\hat{q}}(\xi) \int_{\mathbb{R}^{2}} \frac{\tau_{1}(\xi, \eta)-\tau_{1}(0, \eta)-\left(\tau_{1}(\xi, 0)-\tau_{1}(0,0)\right) \chi(\eta)}{|\eta|^{2}-(|\xi|+i 0)^{2}} \\
& +\dot{\hat{q}}(\xi)\left(\tau_{1}(\xi, 0)-\tau_{1}(0,0)\right) \int_{\mathbb{R}^{2}} \frac{\chi(\eta)}{|\eta|^{2}-(|\xi|+i 0)^{2}} d \eta \\
& -\dot{\hat{q}}(0) \int_{\mathbb{R}^{2}} \frac{\tau_{2}(\xi, \eta)-\tau_{2}(0, \eta)-\left(\tau_{2}(\xi, 0)-\tau_{2}(0,0)\right) \chi(\eta)}{|\eta|^{2}-(|\xi|+i 0)^{2}} d \eta \\
& -\dot{\hat{q}}(0)\left(\tau_{2}(\xi, 0)-\tau_{2}(0,0)\right) \int_{\mathbb{R}^{2}} \frac{\chi\left(\left.\eta\right|^{2}-(|\xi|+i 0)^{2}\right.}{\mid \eta} d \eta \\
= & K_{1}+K_{2}+K_{3}+K_{4}+K_{5} .
\end{aligned}
$$

Applying estimate b) for $A_{1}^{t}$, Theorems 1.1 and 1.4 to $K_{1}$, we see that $K_{1}$ maps $H_{\alpha, N}$ boundedly to $C^{\alpha}(|\xi| \leqq \delta)$. Theorems 1.1 and 1.4 also imply that $K_{2}$ and $K_{4}$ are bounded, and $K_{3}$ and $K_{5}$ are bounded by Lemma 2.3. Thus $J_{3}$ is bounded from $H_{\alpha, N}$ to $C^{\alpha}(|\xi| \leqq \delta)$.

Next we note that the proof of Lemma 2.2 shows that, for $a \in C^{\alpha}(|\xi| \leqq \delta)$, $(a(\xi)-a(0))(\ln |\xi|)^{-1}$ goes to zero in norm in $C^{\alpha}(|\xi| \leqq \delta)$. Since the same conclusion holds if we replace $(\ln |\xi|)^{-1}$ by $g(\xi)(1-f(0,|\xi|))^{-1}$ by the analysis of $(2.10)$, it follows that the $J_{i}, i=2,3$ contribute operators to $T$ whose norm goes to zero as $\delta \rightarrow 0$. The remaining term, $J_{1}$, does not contribute to a term to $T$ whose norm goes to zero with $\delta$, and it will be considered in Proposition 4.2. However, replacing $g(\xi)(1-f(0,|\xi|))^{-1}$ in $J_{1}$ by $\alpha(\ln |\xi|)^{-1}$ only adds a term whose norm goes to zero with $\delta$, when $\alpha=\lim _{|\xi| \rightarrow 0} g(\xi)(1-f(0,|\xi|))^{-1} \ln |\xi|$.

iii) The operator $B_{1}$ is given by

$$
B_{1} \dot{\hat{q}}=A_{1}(\dot{\hat{q}},|\xi|) h_{1}+A_{1}\left(h_{1},|\xi|\right) A_{1}(\dot{\hat{q}},|\xi|) h_{1} .
$$

Thus, since $h_{1}$ comes from $\hat{q} \in C_{0}^{\infty}\left(\mathbb{R}^{2}\right)$, Theorems 1.1 and 1.4 show that $B_{1}$ maps $\dot{\hat{q}}$ boundedly to $C^{\alpha}(|\xi| \leqq \delta)$. Thus by the argument used in ii), we conclude that $T_{1}$ and $T_{8}$ go to zero in norm as $\delta \rightarrow 0$.

iv) As noted earlier, modulo operators of small norm, $\left[T_{0} \dot{\hat{q}}\right](\xi, \eta, k)$ is an integral operator of the form

$$
[V \dot{\hat{q}}](\xi, \zeta, k)=\int_{\mathbb{R}^{2}} \frac{v(\eta, \zeta, k)(\dot{\hat{q}}(\xi-\eta)-\dot{\hat{q}}(-\eta))}{|\eta|^{2}-(k+i 0)^{2}} d \eta,
$$

where $v \in C^{\beta}$ for $\beta<1$ and $v(0, \zeta, k)=0$. Thus

$$
\begin{aligned}
{\left[T_{9} \dot{\hat{q}}\right](\xi,|\xi|)=} & \frac{h_{1}(0,-\xi,|\xi|)}{1-f(0,|\xi|)} m(|\xi|) \int_{\mathbb{R}^{2}} \frac{v(\eta, 0,|\xi|)(\dot{\hat{q}}(\xi-\eta)-\dot{\hat{q}}(-\eta))}{|\eta|^{2}-(|\xi|+i 0)^{2}} d \eta \\
& +\frac{h_{1}(0,-\xi,|\xi|)}{(1-f(0,|\xi|))(2 \pi)^{2}} \int_{\mathbb{R}^{2}} \frac{\dot{\hat{q}}(\xi-\eta)-\dot{\hat{q}}(-\eta)}{|\eta|^{2}-(|\xi|+i 0)^{2}} \\
& \cdot\left(\int_{\mathbb{R}^{2}} \frac{(v(\eta, \zeta,|\xi|)-v(\eta, 0,|\xi|)) \chi(\zeta)}{|\zeta|^{2}-(|\xi|+i 0)^{2}} d \zeta\right) d \eta
\end{aligned}
$$


The first term in (4.14) can be represented as a sum of an integral over $|\xi|<2 \delta$ that is an operator whose norm goes to zero with $\delta$ and of an integral over $|\xi|>2 \delta$ that is clearly a compact operator since $|\xi|<\delta$. Since $v(0, \zeta, k)=0$, the second term in (4.14) also has norm going to zero with $\delta$ by the argument in ii).

v) We claim that $T_{4}$ and $T_{7}$ are also continuous mappings of $H_{\alpha, N}$ to $C^{\alpha}(|\xi| \leqq \delta)$ multiplied by $(1-f(0,|\xi|))^{-1}$ so that they too have norm tending to zero as $\delta \rightarrow 0$. The operators $T_{4}$ and $T_{7}$ are of the form

$$
[U \dot{\hat{q}}](\xi)=g(\xi)\left(1-f(0,|\xi|)^{-2}(I(\xi)-I(0)),\right.
$$

where $g$ is in $C^{\beta}(|\xi| \leqq \delta)$ for all $\beta<1$, and in $T_{4}$,

$$
I(\xi)=(2 \pi)^{-4} \int_{\mathbb{R}^{2}} \int_{\mathbb{R}^{2}} \frac{\chi(\eta) h_{1}(0, \zeta,|\xi|)(\dot{\hat{q}}(\zeta-\eta)-\dot{\hat{q}}(-\eta) \chi(\zeta)-\dot{\hat{q}}(\zeta)+\dot{\hat{q}}(0) \chi(\zeta))}{\left(|\eta|^{2}-(|\xi|+i 0)^{2}\right)\left(|\zeta|^{2}-(|\xi|+i 0)^{2}\right)} d \zeta d \eta .
$$

In $T_{7} I(\xi)$ has the form

$$
I(\xi)=\int_{\mathbb{R}^{2}} \int_{\mathbb{R}^{2}} \frac{u_{1}(\eta, \zeta,|\xi|)(\dot{q}(\zeta-\eta)-\dot{q}(-\eta) \chi(\zeta))+u_{2}(\eta, \zeta,|\xi|) \dot{q}(\zeta)}{\left(|\eta|^{2}-(|\xi|+i 0)^{2}\right)\left(|\zeta|^{2}-(|\zeta|+i 0)\right)^{2}} d \zeta d \eta
$$

where $u_{i}, i=1,2$, is $C^{\beta}$ in all variables for $\beta<1$ and of rapid decrease in $(\eta, \zeta)$. Moreover,

$$
u_{1}(0, \zeta,|\xi|)=u_{2}(0, \zeta,|\xi|)=u_{2}(\eta, 0,|\xi|)=0 .
$$

If we apply the difference operator $\Delta(\mu)$ to $I(\xi)$ in either (4.15) or (4.16) and expand by Liebnitz rule, we can place the difference operator either on the coefficients in the numerators or on one of the two factors in the denominators. If the difference operator is on a coefficient, then the "extra regularity" of the coefficients insures that this term will be bounded by $|\mu|^{\alpha}$. In (4.15) a difference operator on a factor in the denominator gives a term of the form

$$
\begin{aligned}
M(\xi, \mu)= & (2 \pi)^{-2} \int_{\mathbb{R}^{2}}\left[\Delta(\mu)\left(\frac{1}{|\rho|^{2}-(|\xi|+i 0)^{2}}\right)\right] \\
& \cdot\left(\left[A_{1}^{t}(\dot{\hat{q}},|\xi|) g\right](\rho) f_{1}(\rho)-\left[A_{1}^{t}(\dot{\hat{q}},|\xi|) g\right](0) f_{2}(\rho)\right) d \rho,
\end{aligned}
$$

where the $f_{i}$ are $C^{\beta}, \beta<1$, and of fast decrease, and $f_{1}(\rho)=f_{2}(\rho)$ on a neighborhood of $\rho=0$. Combining estimate b) for $A_{1}^{t}$ with Theorem 1.4 , we see that $|M(\xi, \mu)| \leqq$ $C|\mu|^{\alpha}|\ln | \xi||$ as desired. The argument for terms arising from difference operators on the denominator in (4.16) is the same. Thus we have established our claim, and we conclude that the norms of $T_{4}$ and $T_{7}$ go to zero as $\delta \rightarrow 0$.

We are now ready to prove that $d S(\hat{q})=P+T$, where $P$ is invertible and $T P^{-1} T$ is compact. The preceding remarks have reduced this to the following propositions:

Proposition 4.1. For any $\rho(|\xi|) \in C_{0}^{\infty}\left(\mathbb{R}^{2}\right)$ satisfying $\rho(|\xi|)=1$ on a neighborhood of $\xi=0$, the operator

$$
(1-\rho)(d S(\hat{q})-P) P^{-1}(1-\rho)(d S(\hat{q})-P)
$$

is compact from $H_{\alpha, N}$ to $H_{\alpha, N}$ for $\alpha<\frac{1}{2}, N>0$. 
Proposition 4.2. Let $\rho$ be as in Proposition 4.1 and let $W$ be given by

$$
[W \dot{\hat{q}}](\xi)=\frac{1}{\ln |\xi|} \int_{\mathbb{R}^{2}}\left(\frac{\dot{\hat{q}}(\xi-\eta)-\chi(\eta) \dot{\hat{q}}(0)}{|\eta|^{2}-(|\xi|+i 0)^{2}}-\frac{\dot{\hat{q}}(-\eta)-\chi(\eta) \dot{\hat{q}}(0)}{|\eta|^{2}}\right) d \eta .
$$

Then $\rho W P^{-1} \rho W$ restricted to the subspace $\stackrel{\circ}{H}_{\alpha N}=\left\{f \in H_{\alpha, N}: f(0)=0\right\}$ has norm tending to zero as the support of $\rho$ tends to $\xi=0$.

Proposition 4.3. The product of $(1-\rho)(d S(\hat{q})-P) P^{-1} \rho$ with the operator $W$ of Proposition 4.2 is compact.

The proof of Proposition 4.1 is the argument given in Sect. 4 of [ER]. There is one significant difference between two and three dimensions. When one carries out the computations to determine the singularities of the kernel of $((1-\rho)(d S-P)) P^{-1}((1-\rho)(d S-P))$ one is lead, as in [ER, pp. 203-204], to integrals of the form

$$
I(\xi, \tau)=\int_{\mathbb{R}^{p}} \frac{f\left(\mu_{2}, \xi, \tau\right) d \mu_{2}}{\sqrt{|\xi|^{2}-\left|\mu_{2}\right|^{2}}\left(|\tau|-\frac{\xi \cdot \tau}{|\tau|}-\sqrt{|\xi|^{2}-\left|\mu_{2}\right|^{2}}-i 0\right)} .
$$

However, we now have $p=1$ instead of 2 as in [ER]. Substituting $\mu_{2}=$ $\omega\left(2|\xi|-\omega^{2}\right)^{1 / 2}$, one sees

$$
I(\xi, \tau)=\int_{\mathbb{R}} \frac{2 f\left(\omega\left(2|\xi|-\omega^{2}\right)^{1 / 2}, \xi \cdot \tau\right) d \omega}{\sqrt{2|\xi|-\omega^{2}}\left(|\tau|-\frac{\xi \cdot \tau}{|\tau|}-|\xi|+\omega^{2}-i 0\right)},
$$

where $\omega^{2}<|\xi|$ on the support of $f$. Thus

$$
\begin{aligned}
I(\xi, \tau) & =\left(\frac{2}{|\xi|}\right)^{1 / 2} f(0, \xi, \tau) \int_{\mathbb{R}} \frac{d \omega}{|\tau|-\frac{\xi \cdot \tau}{|\tau|}-|\xi|+\omega^{2}-i 0}+I_{0}(\xi, \tau) \\
& =\left(\frac{2}{|\xi|}\right)^{1 / 2} f(0, \xi, \tau) \frac{\pi}{\sqrt{\left(|\tau|-\frac{\xi \cdot \tau}{|\tau|}-|\xi|-i 0\right)}}+I_{0}(\xi \cdot \tau),
\end{aligned}
$$

where $\sqrt{ }$ is the branch positive for positive argument and $I_{0}(\xi, \tau)$ has a milder singularity. The term $I_{0}$ actually behaves like the leading singular term in [ER] with a singularity like $\ln \left(|\tau|-\frac{\xi \cdot \tau}{|\tau|}-|\xi|-i 0\right)$. The first term in (4.18) is an integral kernel mapping $H_{\alpha, N}$ into $C^{\alpha^{\prime}}$ for any $\alpha^{\prime}<\frac{1}{2}$. For this reason we need to assume $\alpha<\frac{1}{2}$ in Proposition 4.1. The remainder of the proof of Proposition 4.1 is the same as the argument of [ER, Sect. 4], and we will not give it here.

Proposition 4.2 is the most delicate of these three propositions, and the most tedious to prove. This is the argument which we will give here. The proof of Proposition 4.3 is simpler, and will be omitted. 
On $\stackrel{\circ}{H, N}_{\alpha, ~}$ the integral operator $-(\rho W) P^{-1}(\rho W)$ has the kernel

$$
\begin{aligned}
K(\xi, \tau)= & \lim _{\varepsilon_{2} \downarrow 0} \lim _{\varepsilon_{1} \downarrow 0} \frac{\rho(|\xi|)}{\ln |\xi|} \int_{\mathbb{R}^{2}}\left(\frac{4}{|\xi-2 \eta|^{2}-\left(|\xi|+i \varepsilon_{2}\right)^{2}}-\frac{1}{|\eta|^{2}}\right) \\
& \cdot \frac{\rho(|\eta|)}{\ln |\eta|}\left(\frac{1}{|\tau-\eta|^{2}-\left(|\eta|+i \varepsilon_{1}\right)^{2}}-\frac{1}{|\tau|^{2}}\right) d \eta,
\end{aligned}
$$

where the limits are taken in distribution sense. As in [ER] our objective is to analyze the singularities of $K(\xi, \tau)$. We will show, for $\alpha \leqq 1 / 2$, that they are sufficiently mild that $\ln |\xi| K(\xi, \tau)$ maps $\stackrel{\circ}{H}_{\alpha, N}$ boundedly to $C^{\alpha}(|\xi| \leqq \delta)$.

To study the behavior of $K(\xi, \tau)$ near $(\xi, \tau)=(0,0)$ we will stretch variables by setting $\eta=r \eta^{\prime}, \xi=r \zeta^{\prime}$ and $\tau=r \tau^{\prime}$, where $r=\left(|\xi|^{2}+|\tau|^{2}\right)^{1 / 2}$. This gives

$$
K(\xi, \tau)=\frac{\rho(|\xi|)|\xi|}{r^{2}|\tau| \ln |\xi|} I(\xi, \tau)
$$

where, using $\hat{v}$ for the unit vector in direction of $v$,

$$
I(\xi, \tau)=\int_{\mathbb{R}^{2}} \frac{8(\hat{\eta} \cdot \hat{\xi})(\hat{\eta} \cdot \hat{\tau}) \rho\left(r\left|\eta^{\prime}\right|\right) d \eta^{\prime}}{\left(\left|\xi^{\prime}-2 \eta^{\prime}\right|^{2}-\left(\left|\xi^{\prime}\right|+i 0\right)^{2}\right) \ln \left(r\left|\eta^{\prime}\right|\right)\left(\left|\tau^{\prime}-\eta^{\prime}\right|^{2}-\left(\left|\eta^{\prime}\right|+i 0\right)^{2}\right.} .
$$

For $\delta|\xi|<|\tau|<\delta^{-1}|\xi|$ the zeros of the denominator of the integrand of $I(\xi, \tau)$ are bounded away from $\eta^{\prime}=0$. Moreover, excluding a neighborhood of $\eta^{\prime}=0, I\left(\xi^{\prime}, \tau^{\prime}\right)$ has the form of the kernel of the operator $T_{1}$ defined in (4.9) of [ER] with $2 \tau$ replaced by $\tau$. Thus, for $\delta|\xi|<|\tau|<\delta^{-1}|\xi|$, it follows from (4.18) that $I(\xi, \tau)=$ $\hat{\xi} \cdot A\left(\xi^{\prime}, \tau^{\prime}, r\right) \hat{\tau}$ with the matrix $A$ given by

$$
A\left(\xi^{\prime}, \tau^{\prime}, r\right)=\frac{h_{0}\left(\xi^{\prime}, \tau, \ln r, r\right)}{\sqrt{\left|\xi^{\prime}\right|} \sqrt{\left|\tau^{\prime}\right|-\frac{\xi^{\prime} \cdot \tau^{\prime}}{\left|\tau^{\prime}\right|}-\left|\xi^{\prime}\right|-i 0}}+A_{0}\left(\xi^{\prime}, \tau^{\prime}, \ln r, r\right)+A_{1}\left(\xi^{\prime}, \tau^{\prime}, \ln r, r\right),
$$

where $h_{0}$ is smooth and order $|\ln r|^{-1}, A_{0}$ is a superposition of functions of the form

$$
h_{1}\left(\xi^{\prime}, \tau^{\prime}, \ln r, r\right) \ln \left(\left|\tau^{\prime}\right|-\frac{\xi^{\prime} \cdot \tau^{\prime}}{\left|\tau^{\prime}\right|}-\sqrt{\left|\xi^{\prime}\right|^{2}-a}-i 0\right)
$$

where $h_{1}$ is smooth and order $(\ln r)^{-1}, a \leqq \frac{1}{2}\left|\xi^{\prime}\right|$, and $A_{1}$ is $C^{\alpha^{\prime}}$ for all $\alpha^{\prime}<1$ in $\xi^{\prime}$ and order $(\ln r)^{-1}$. Note that for $\delta|\xi|<|\tau|<\delta^{-1}|\xi|$ both $\left|\xi^{\prime}\right|$ and $\left|\tau^{\prime}\right|$ lie in $[\varepsilon, 1-\varepsilon]$ with $\varepsilon=\varepsilon(\delta)>0$.

When $|\tau|>2|\xi|$ the zeros of $\left|\xi^{\prime}-2 \eta^{\prime}\right|^{2}=\left|\xi^{\prime}\right|^{2}$ and $\left|\tau^{\prime}-\eta^{\prime}\right|^{2}-\left|\eta^{\prime}\right|^{2}$ are disjoint. Thus, taking $\left|\xi^{\prime}\right|<1 / 8$, we can use a cutoff $\chi\left(\left|\eta^{\prime}\right|\right)$ to split $I(\xi, \tau)$ into

$$
I_{2}(\xi, \tau)=\int_{\mathbb{R}^{2}} \frac{(\hat{\eta} \cdot \hat{\xi})(\hat{\eta} \cdot \hat{\tau}) g\left(\eta^{\prime}, \xi^{\prime}, \tau^{\prime}\right) \chi\left(\left|\eta^{\prime}\right|\right)}{\left(\left|\eta^{\prime}\right|^{2}-\xi^{\prime} \cdot \eta^{\prime}-i 0\right) \ln \left(r\left|\eta^{\prime}\right|\right)} d \eta^{\prime},
$$

where $g$ is smooth and bounded, and

$$
I_{3}(\xi, \tau)=\int_{\mathbb{R}^{2}} \frac{(\hat{\eta} \cdot \hat{\xi})(\hat{\eta} \cdot \hat{\tau}) h\left(\eta^{\prime}, \xi^{\prime}\right)\left(1-\chi\left(\left|\eta^{\prime}\right|\right) \rho\left(\left|\eta^{\prime}\right| r\right)\right.}{\left(\left|\tau^{\prime}\right|^{2}-2 \tau^{\prime} \cdot \eta^{\prime}-i 0\right) \ln r\left|\eta^{\prime}\right|} d \eta^{\prime}
$$

where $h$ is smooth, and $\left|\partial_{\eta^{\prime}}^{\alpha} h\right| \leqq C\left|\eta^{\prime}\right|^{-2-|\alpha|}$. Clearly $I_{3}$ is of the form $\hat{\xi} \cdot A\left(\tau^{\prime}, \xi^{\prime}, \ln r\right) \hat{\tau}$, 
where $A$ is a smooth matrix-valued function which is $O\left((\ln r)^{-1}\right)$. In $I_{2}$ we split $g\left(\eta^{\prime}, \xi^{\prime}, \tau^{\prime}\right)$ into $g\left(0, \xi^{\prime}, \tau^{\prime}\right)$ and $g\left(\eta^{\prime}, \xi^{\prime}, \tau^{\prime}\right)-g\left(0, \xi^{\prime}, \tau^{\prime}\right)$, forming $I_{21}$ and $I_{22}$. In $I_{21}$ we use polar coordinates centered at $\eta^{\prime}=0$. Since this gives $\hat{\eta} \cdot \hat{\xi}=\cos \varphi$ and $\hat{\eta} \cdot \tau^{\prime}=$ $\cos \left(\varphi-\varphi_{0}\right)$, where $\varphi_{0}$ is the angle counterclockwise from $\xi$ to $\tau$, one can evaluate the integral in $\varphi$ explicitly:

$$
I_{21}(\xi, \tau)=\frac{\pi g\left(0, \xi^{\prime}, \tau^{\prime}\right)}{2} \int_{0}^{\infty}\left[\frac{s \cos \varphi_{0}\left(s-\sqrt{\left.s^{2}-\left|\xi^{\prime}\right|^{2}-i 0\right)}\right.}{\left|\xi^{\prime}\right|^{2} \sqrt{s^{2}-\left|\xi^{\prime}\right|^{2}-i 0}}\right] \frac{\chi(s) d s}{\ln r s} .
$$

Setting $s=\left|\xi^{\prime}\right| u$, we have

$$
\begin{aligned}
I_{21}(\xi, \tau) & =\frac{\pi g\left(0, \xi^{\prime}, \tau^{\prime}\right)}{2} \hat{\tau} \cdot \hat{\xi} \int_{0}^{\infty}\left(\frac{u\left(u-\sqrt{\left.u^{2}-1-i 0\right)}\right.}{\sqrt{u^{2}-1-i 0}}\right) \frac{\chi\left(\left|\xi^{\prime}\right| u\right)}{\ln \left(r\left|\xi^{\prime}\right| u\right)} d u \\
& =\pi g\left(0, \xi^{\prime}, \tau^{\prime}\right) \hat{\tau} \cdot \hat{\xi}\left[\int_{i}^{\infty} \frac{1}{u} \frac{\chi\left(\left|\xi^{\prime}\right| u\right)}{\ln \left(r\left|\xi^{\prime}\right| u\right)} d u+I_{21}^{\prime}\right],
\end{aligned}
$$

where $I_{21}^{\prime}=\int_{0}^{\infty} f(u) \frac{\chi\left(\left|\xi^{\prime}\right| u\right)}{\ln \left(r\left|\xi^{\prime}\right| u\right)} d u$ with $\int_{0}^{\infty}|f(u)| d u<\infty$. Assuming $\chi(s)=1$ for $|s|<\delta$,

$$
\int_{1}^{\infty} \frac{1}{u} \frac{\chi\left(\left|\xi^{\prime}\right| u\right)}{\ln \left(r\left|\xi^{\prime}\right|\right) u} d u=\int_{\delta}^{\infty} \frac{1}{v} \frac{\chi(v) d v}{\ln r v}+\left.\ln |\ln r v|\right|_{\left|\xi^{\prime}\right|} ^{\delta} .
$$

Since $\left|g\left(\eta^{\prime}, \xi^{\prime}, \tau^{\prime}\right)-g\left(0, \xi^{\prime}, \tau^{\prime}\right)\right| \leqq C\left|\eta^{\prime}\right|, I_{22}$ is less singular than $I_{21}$.

The remaining case is $|\tau|<\delta|\xi|, \delta$ small. The new feature of this case is that the factors in the denominator of the integrand have common zeros near $\eta^{\prime}=0$. Since common zeros outside $\left|\eta^{\prime}\right|<\varepsilon(\delta)$ occur when the zero sets of the factors intersect transversally and hence contribute smooth functions of $\left(\xi^{\prime}, \tau^{\prime}, \ln r, \hat{\xi}, \hat{\tau}\right)$, we will only consider the integrand multiplied by a cutoff $\chi\left(\left|\eta^{\prime}\right|\right)$ supported in the set where $\rho\left(r\left|\eta^{\prime}\right|\right) \equiv 1$ for $r<1$. Thus the contribution to $I(\xi, \tau)$ is of the form

$$
J(\xi, \tau)=\frac{1}{\left|\tau^{\prime}\right|} \int_{0}^{\infty} \int_{0}^{2 \pi} \frac{8 \cos \varphi \cos \left(\varphi-\varphi_{0}\right) \chi(s)}{\left(s-\left|\xi^{\prime}\right| \cos \varphi-i 0\right)(\ln r s)\left(\left|\tau^{\prime}\right|-2 s \cos \left(\varphi-\varphi_{0}\right)-i 0\right)} d \varphi d s,
$$

where we have again used the polar coordinates $s=\left|\eta^{\prime}\right|, \hat{\eta} \cdot \widehat{\xi}=\cos \varphi, \hat{\eta} \cdot \hat{\tau}=\cos \left(\varphi-\varphi_{0}\right)$. As one would expect, the function $J(\xi, \tau)$ is most singular when the zero sets of the factors of the denominator of the integrand are tangent, i.e. when

$$
\left|\xi^{\prime}\right|\left(1+\cos \varphi_{0}\right)=\left|\tau^{\prime}\right| \text {. }
$$

To study this singularity one can evaluate the integral in $\varphi$ (the method of residues is convenient here), and then study the integral in $s$. This contains spurious singularities at $s=\left|\xi^{\prime}\right|$ and $2 s=\left|\tau^{\prime}\right|$ that can be eliminated by changes of variable, and more complicated spurious singularities when $\left|\tau^{\prime}\right|=2\left|\xi^{\prime}\right| \cos \varphi_{0}$. Instead of tracking down the cancellations which eliminate the latter singularities, we simply split this case into two, considering first $|\tau|<\delta|\xi|,\left|\cos \varphi_{0}\right|>1-\delta$, where these singularities do not occur. In this case the result is that

$$
J=\frac{1}{\left|\tau^{\prime}\right|} \frac{f}{\sqrt{b-i 0}}
$$


modulo less singular terms. Here $b=\left|\xi^{\prime}\right|^{2}-\left(\left|\tau^{\prime}\right|-\left|\xi^{\prime}\right| \cos \varphi_{0}\right)^{2}$ and $f$ is a smooth, bounded function of $\xi^{\prime}, \tau^{\prime}, \varphi_{0}, \sqrt{b-i 0}, \ln r$ and the functions $\ln \left( \pm\left|\xi^{\prime}\right| \sin \varphi_{0} \pm\right.$ $\sqrt{b-i 0)}$.

When $|\hat{\xi} \cdot \hat{\tau}|<1-\delta$, the functions $u=2 \hat{\tau} \cdot \eta^{\prime}$ and $v=\xi^{\prime} \cdot \eta^{\prime}-\left|\eta^{\prime}\right|^{2}$ can be used as coordinates near $\eta^{\prime}=0$. Thus, assuming $|\tau|<\delta|\xi|$ and taking the support of $\chi$ sufficiently small, for $(\xi, \tau)$ in this region we have

$$
J(\xi, \tau)=\frac{1}{\left|\tau^{\prime}\right|\left|\xi^{\prime}\right|} \int_{\mathbb{R}^{2}} \frac{u(v+g(u, v)) f(u, v) d u d v}{(v-i 0)\left(\left|\tau^{\prime}\right|-u-i 0\right)\left(\ln r^{2} g(u, v)\right) g(u, v)},
$$

where $g(u, v)=\left|\eta^{\prime}\right|^{2}$ and $f$ is smooth with support near $(u, v)=(0,0)$. Thus

$$
\begin{aligned}
J(\xi, \tau)= & \frac{1}{\left|\tau^{\prime}\right|\left|\xi^{\prime}\right|} \int_{\mathbb{R}^{2}} \frac{I_{1}\left(u, \xi^{\prime}, \hat{\tau}, \ln r\right)}{\left(\left|\tau^{\prime}\right|-u-i 0\right)} d u \\
& +\frac{1}{\left|\tau^{\prime}\right|\left|\xi^{\prime}\right|} \int_{\mathbb{R}^{2}} \frac{I_{2}\left(u, \xi^{\prime}, \hat{\tau}, \ln r\right)}{\left(\left|\tau^{\prime}\right|-u-i 0\right)} d u \equiv J_{1}+J_{2},
\end{aligned}
$$

where

$$
I_{1}\left(u, \xi^{\prime}, \hat{\tau}, \omega\right)=\int_{\mathbb{R}} \frac{u f(u, v)}{(2 \omega+\ln g(u, v)) g(u, v)} d v
$$

and

$$
I_{2}\left(u, \xi^{\prime}, \hat{\tau}, \omega\right)=\int_{\mathbf{R}} \frac{u f(u, v)}{(v-i 0)(2 \omega+\ln g(u, v))} d v .
$$

Note that $f$ and $g$ depend smoothly on $\xi^{\prime}$ and $\hat{\tau}$.

To check the regularity of $I_{2}$ in $u$ we set $v=u s$. This gives

$$
I_{2}=\int_{\mathbb{R}} \frac{u f(u, u s) d s}{(s-(\operatorname{sgn} u) i 0)(2 \omega+\ln g(u, s u))} .
$$

Since we may assume that $g(u, v)<\frac{1}{2}$ on support $f$, one sees that $I_{2}$ is $C^{1-\varepsilon}$ in $u$ uniformly on $\omega \leqq 0$. Moreover, $\left|I_{2}\right|<C|\omega|^{-1}$ and $\left|\frac{\partial I_{2}}{\partial \omega}\right| \leqq C|\omega|^{-2}$ for $\omega<0$. Applying Privaloff's theorem, we may conclude that $J_{2}=\left|\tau^{\prime}\right|^{-1} F_{2}\left(\xi^{\prime}, \hat{\tau},\left|\tau^{\prime}\right|, \ln r\right)$, where $F_{2}$ is smooth in $\left(\xi^{\prime}, \hat{\tau}, \ln r\right)$ and $C^{1-\varepsilon}$ in $\left|\tau^{\prime}\right|$, and $F_{2}\left(\xi^{\prime}, \hat{\tau},\left|\tau^{\prime}\right|, \omega\right)$ satisfies the same estimates as $I_{2}$ as $\omega \rightarrow \infty$.

$I_{1}$ is a little more singular. We will write

$$
\left|\tau^{\prime}\right|\left|\xi^{\prime}\right| J_{1}=I_{1}\left(\left|\tau^{\prime}\right|\right) \int_{\mathbf{R}} \frac{\chi(u) d u}{\left|\tau^{\prime}\right|-u-i 0}+\int_{\mathbf{R}} \frac{\left(I_{1}(u)-I_{1}\left(\left|\tau^{\prime}\right|\right)\right) \chi(u)}{\left|\tau^{\prime}\right|-u} d u
$$

where $\chi$ is a smooth cutoff, equal 1 on support $f$.

Since $u \frac{\partial g}{\partial u} g^{-1}$ is bounded, one computes that $\partial I_{1} / \partial u$ is bounded uniformly by 
$C|u|^{-1}$. We also have

$$
\int_{\mathbb{R}} \frac{\left(I_{1}(u)-I_{1}\left(\left|\tau^{\prime}\right|\right)\right) \chi(u)}{\left|\tau^{\prime}\right|-u} d u=\int_{\mathbb{R}} \frac{I_{1}\left(\left|\tau^{\prime}\right| y\right)-I_{1}\left(\left|\tau^{\prime}\right|\right)}{1-y} \chi\left(\left|\tau^{\prime}\right| y\right) d y
$$

so that

$$
\frac{\partial}{\partial\left|\tau^{\prime}\right|}\left(\int_{\mathbb{R}} \frac{\left(I_{1}(u)-I_{1}\left(\left|\tau^{\prime}\right|\right)\right) \chi(u)}{\left|\tau^{\prime}\right|-u} d u\right)
$$

is bounded by $\frac{C}{\left|\tau^{\prime}\right|}$. Thus we conclude that

$$
\left|\tau^{\prime}\right|\left|\xi^{\prime}\right| J=F_{1}\left(\xi^{\prime}, \tau,\left|\tau^{\prime}\right|\right)+F_{2}\left(\xi^{\prime}, \tau,\left|\tau^{\prime}\right|\right)
$$

where $F_{i}$ is smooth in $\xi^{\prime}$ and bounded in $\tau, F_{2}$ is $C^{1-\varepsilon}$ in $\left|\tau^{\prime}\right|$ uniformly in $\left(\xi^{\prime}, \tau\right)$, and $\partial F_{1} / \partial\left|\tau^{\prime}\right|$ is bounded by $C\left|\tau^{\prime}\right|^{-1}$.

The final point to be checked here is that the singularities we have found are sufficiently mild that $(\ln |\xi|)(\rho W) P^{-1}(\rho W)$ is a bounded map of

$$
\stackrel{\circ}{H}_{\alpha, N}=\left\{v \in H_{\alpha, N}: r(0)=0\right\} \quad \text { to } \quad \stackrel{\circ}{C}^{\alpha}=\left\{v \in C^{\alpha}(|\xi| \leqq 1): v(0)=0\right\} .
$$

Hence $(\rho W) P^{-1}(\rho W)$ can be given arbitrarily small norm by taking the support of $\rho$ sufficiently close to zero.

The list of singularities we have found is as follows. When $\delta|\xi|<|\tau|<\delta^{-1}|\xi|$, the most singular terms are of the form

$$
K_{1}(\xi, \tau)=\frac{\rho(\xi) \xi \cdot f}{r|\xi|^{1 / 2}|\tau| \ln |\xi|(|\tau|-\xi \cdot \hat{\tau}-|\xi|-i 0)^{1 / 2}}
$$

where $f$ is smooth in $(\xi / r, \tau / r, \ln r, r)$ and bounded in $\tau$. Moreover, $f$ is order $|\ln r|^{-1}$ as $r \rightarrow 0$. When $4|\xi|<|\tau|$, the most singular terms are of the form

$$
K_{2}(\xi, \tau)=\frac{\rho(|\xi|) \xi \cdot f \ln (\ln |\xi|)^{-1}}{r^{2}|\tau| \ln |\xi|}
$$

where $f$ is a smooth function of $(\xi / r, \tau / r)$, bounded in $\tau$. When $\delta|\xi|>|\tau|$ and $|\hat{\xi} \cdot \hat{\tau}|>(1-\delta)$ with $\delta$ sufficiently small, the most singular terms are of the form

$$
K_{3}(\xi, \tau)=\frac{\rho(|\xi|)|\xi| f}{(\ln |\xi|)|\tau|^{2} \sqrt{|\xi|^{2}-(|\tau|-\xi \cdot \hat{\tau})^{2}-i 0}}
$$

where $f$ is as in (4.23). form

Finally, when $\delta|\xi|>|\tau|$ and $|\hat{\xi} \cdot \hat{\tau}|<(1-\delta)$ the most singular terms are of the

$$
K_{4}(\xi, \tau)=\frac{\sigma(|\xi|)}{|\tau|^{2} \ln |\xi|} f(\xi / r, \tau / r, \hat{\tau})
$$


where $f(u, v, w)$ is smooth in $u$, bounded in $w$ and $\partial f / \partial v$ is bounded by $|v|^{-1}$.

We will introduce a partition of unity subordinate to the cover of $\left\{\left(\xi^{\prime}, \tau^{\prime}\right):\left|\xi^{\prime}\right|^{2}+\right.$ $\left.\left|\tau^{\prime}\right|^{2}=1\right\}$ by

$$
\left\{\delta\left|\xi^{\prime}\right|<\left|\tau^{\prime}\right|<\delta^{-1}\left|\xi^{\prime}\right|\right\}, \quad\left\{\left|\xi^{\prime}\right|<2 \delta\left|\tau^{\prime}\right|\right\} \quad \text { and }\left\{\left|\tau^{\prime}\right|<2 \delta\left|\xi^{\prime}\right|\right\}
$$

as well as a partition of unity subordinate to the cover of $\left\{(\hat{\xi}, \hat{\tau}):\left|\hat{\xi}^{\prime}\right|=|\hat{\tau}|=1\right\}$ by

$$
\{|\hat{\xi} \cdot \hat{\tau}|<(1-2 \delta)\} \quad \text { and } \quad\{\hat{\xi} \cdot \hat{\tau} \mid>(1-\delta)\} \text {. }
$$

Then the leading singularity in $K(\xi, \tau)$ is the sum of $K_{1}, K_{2}, K_{3}$ and $K_{4}$ multiplied by the appropriate factors from the partitions of unity. Since this only introduces additional factors of smooth functions of $\left(\xi^{\prime}, \tau^{\prime}\right)$ in $K_{1}$ and $K_{2}$ and smooth functions of $\left(\xi^{\prime}, \tau^{\prime}, \hat{\xi} \cdot \hat{\tau}\right)$ in $K_{2}$ and $K_{3}$, the only change in the forms of the singularities is that $f$ in (4.28) now depends on $\hat{\xi} \cdot \hat{\tau}$ as well as $(\xi / r, \tau / r, \hat{\tau})$.

The kernel $K_{3}(\xi, \tau)$ of $(4.27)$ has the most complicated behavior. To estimate

$$
\sup _{|\mu|<1 / 2|\xi|,|\xi|<1}\left|\int_{\mathbb{R}^{2}} \Delta(\mu)\left(\ln |\xi| K_{3}(\xi, \tau)\right) f(\tau) d \tau\right|,
$$

it will suffice to consider $\Delta(\mu)$ acting on each factor in $(\ln |\xi|) K_{3}$ separately. Letting $\Delta(\mu)$ act on $(|\xi|+\xi \cdot \hat{\tau}-|\tau|-i 0)^{-1 / 2}$, and setting $|\tau|=s, \hat{\xi} \cdot \hat{\tau}=\cos \varphi$, we get a term bounded by

$$
C|\xi| \int_{0}^{2 \delta|\xi|} \int_{|\cos \varphi|>1-2 \delta} \frac{s^{-1}}{(|\xi|(1-\cos \varphi)+s)^{1 / 2}}\left|\left(\Delta(\mu)(|\xi|+\xi \cdot \hat{\tau}-s-i 0)^{-1 / 2}\right) f(\tau) d \varphi\right| d s .
$$

It suffices to estimate (4.29) separately when $\varphi_{0} \sim 0$ and $\varphi_{0} \sim \pi$ and to consider only $\mu$ giving purely radial or purely rotational changes in $\xi$. This gives us four cases: a) $\mu \| \xi$ and $\cos \varphi<2 \delta-1$, b) $\mu \| \xi$ and $\cos \varphi>1-2 \delta$, c) $|\xi+\mu|=|\xi|$ and $\cos \varphi<2 \delta-1$, and d) $|\xi+\mu|=|\xi|$ and $\cos \varphi>1-2 \delta$.

Since for $f \in \stackrel{\circ}{\alpha, N}_{\alpha},|f(\tau)| \leqq|\tau|^{\alpha}\|f\|_{\alpha, N}$, in case a) (4.29) is bounded by

$$
\begin{aligned}
C|\xi|^{1 / 2}\|f\|_{\alpha, N} \int_{0}^{2 \delta /|\xi|} s^{\alpha-1} \int_{\pi-\delta^{\prime}}^{\pi+\delta^{\prime}} \\
\\
\left|(|\xi+\mu|(1+\cos \varphi)-s-i 0)^{-1 / 2}-(|\xi|(1+\cos \varphi)-s-i 0)^{-1 / 2}\right| d \varphi d s,
\end{aligned}
$$

or, setting $s=|\xi| u$,

$$
\begin{aligned}
& C|\xi|^{\alpha}\|f\|_{\alpha, N} \int_{0}^{2 \delta} u^{\alpha-1} \int_{\pi-\delta^{\prime}}^{\pi+\delta^{\prime}} \\
& \left|\left(|\xi+\mu \| \xi|^{-1}(1+\cos \varphi)-u-i 0\right)^{-1 / 2}-(1+\cos \varphi-u-i 0)^{-1 / 2}\right| d \varphi d u .
\end{aligned}
$$

Setting $(1+\cos \varphi)=u \beta^{2}$, i.e. $u^{1 / 2} \beta=\sqrt{2} \cos \frac{\varphi}{2}$, we see that $(4.30)$ is bounded by

$$
\left.C|\xi|^{\alpha}\|f\|_{a, N} \int_{0}^{2 \delta} u^{\alpha-1} \int_{-c / \sqrt{u}}^{c / \sqrt{u}} \mid(1+\Delta) \beta^{2}-1-i 0\right)^{-1 / 2}-\left(\beta^{2}-1-i 0\right)^{-1 / 2} \mid d \beta d u,
$$

where $\Delta=|\xi+\mu \| \xi|^{-1}-1$. We have $|\Delta| \leqq|\mu||\xi|^{-1}$, and, since $|\mu|<\frac{1}{2}|\xi|,|\Delta|<\frac{1}{2}$. 
To estimate the inner integral we split it into the integrals over $\{|1-\beta|<|\Delta|\}$ and $\{|1-\beta|>|\Delta|\}$. Estimating the integrals of $\left|(1+\Delta) \beta^{2}-1\right|^{-1 / 2}$ and $\left|\beta^{2}-1\right|^{-1 / 2}$ separately over the first domain, and then using the cancellation in the integrand in the second domain, we see that (4.31) is bounded by

$$
C\left(\frac{|\mu|}{|\xi|}\right)^{1 / 2}|\xi|^{\alpha}\|f\|_{\alpha, N}
$$

and, since $|\mu|<\frac{1}{2}|\xi|$, this is bounded by $|\mu|^{\alpha}\|f\|_{\alpha, N}$ as desired.

In case b) (4.29) is bounded by

$$
\frac{C|\mu|\|f\|_{\alpha, N}}{|\xi|^{1 / 2}} \int_{0}^{2 \delta|\xi|}\left(\int_{0}^{\delta \prime} \frac{s^{\alpha-1} d \varphi}{(|\xi|(1-\cos \varphi)+s)^{1 / 2}}\right) d s \leqq \frac{C \mid \mu\|f\|_{\alpha, N}}{|\xi|^{1-\alpha}}
$$

which, since $|\mu|<\frac{1}{2}|\xi|$, is stronger than(4.32).

In case c) the change of variables that lead to (4.30) shows that (4.29) is bounded by

$$
\begin{aligned}
& \left.C \int_{0}^{2 \delta} u^{-1}\right|_{|\pi-\varphi|<\delta^{\prime}}\left((1+\cos (\varphi-\varphi(\mu))-u-i 0)^{-1 / 2}\right. \\
& \left.\quad-(1+\cos \varphi-u-i 0)^{-1 / 2}\right) f(|\xi| u \cos \varphi,|\xi| u \sin \varphi) d \varphi \mid d u,
\end{aligned}
$$

where $2|\xi| \mid \sin \left(\varphi(\mu) / 2|=| \mu \mid\right.$. Writing the integral over $|\pi-\varphi|<\delta^{\prime}$ as the sum of the integrals of the two terms in the integrand, sending $\varphi-\varphi(\mu)$ to $\varphi$ in the first and recombining, one sees that (4.33) is bounded by

$$
|\mu|^{\alpha}\|f\|_{\alpha, N} \int_{0}^{2 \delta} u^{-1+\alpha} \int|1+\cos \varphi-u|^{-1 / 2} d \varphi d u .
$$

Since the integral is finite, we again have the desired estimate.

Case d) is a simpler version of c), just as b) was a simpler version of a).

When $\Delta(\mu)$ acts on $(|\xi|-\xi \cdot \hat{\imath}+|\tau|)^{-1 / 2}$ one encounters terms like those we have just estimated. The remaining factors are less singular and do not cause problems.

To estimate

$$
\sup _{1 / 2|\xi|<|\mu|<2}\left|\int_{\mathbb{R}^{2}} \Delta(\mu)\left(\ln |\xi| K_{3}(\xi, \tau)\right) f(\tau) d \tau\right|,
$$

we simply note that

$$
\int_{\mathbb{R}^{2}}|\ln | \xi\left|K_{3}(\xi, \tau)\right||\tau|^{\alpha} d \tau \leqq C|\xi|^{\alpha} \int_{0}^{\delta} u^{\alpha-1} \int_{|\cos \varphi|>1-2 \delta}\left|1-(u-\cos \varphi)^{2}\right|^{-1 / 2} d \varphi d s \leqq C|\xi|^{\alpha} .
$$

Thus

$$
\left|\int_{\mathbb{R}^{2}} \Delta(\mu)\left(\ln |\xi| K_{3}(\xi, \tau)\right) f(\tau) d \tau\right| \leqq C\left(|\mu+\xi|^{\alpha}+|\xi|^{\alpha}\right)\|f\|_{\alpha, N}
$$

and, since $|\xi|<2|\mu|$, we have the desired estimate.

The preceding analysis should be repeated for $K_{1}, K_{2}$, and $K_{4}$. As noted earlier these are somewhat less singular. 


\section{Conclusion}

From the arguments of Sects. 3 and 4 it follows that the Frechet derivative $d S(\hat{q})$ is a Fredholm operator of index zero for $\hat{q}$ in a connected component $\mathscr{U}_{0}$ of $\mathscr{U}$ such that $\mathscr{S}_{0}=\mathscr{U}_{0} \cap\left\{\hat{q}: q \in C_{0}^{\infty}\left(\mathbb{R}^{2}\right)\right.$ and $q$ is real-valued $\}$ is dense in $H_{\alpha, N}^{r}$. To complete the proof of the result stated in the Introduction we prove the following lemma.

Lemma 5.1. The Frechet derivative $d S(\hat{q})$ is invertible for some $\hat{q}_{0} \in \mathscr{U}_{0}$, and hence, by the analyticity of $S(\hat{q})$ on $\mathscr{U}_{0}$, for all $\hat{q}$ in an open dense subset of $\mathscr{U}_{0}$.

Proof. We will show that there are $\hat{q}_{0} \in \mathscr{S}_{0}$ with small norm in $H_{\alpha, N}$ such that $d S\left(\hat{q}_{0}\right)$ is invertible. Using the decomposition $d S=P+T$ of Sect. 4 , it suffices to show that $\left(I-T P^{-1}\right) d S=P-T P^{-1} T$ is invertible, and we will do this by showing that for a suitably chosen sequence $\hat{q}_{n} \in \mathscr{S}_{0}$ converging to zero, the norm of $T\left(\hat{q}_{n}\right) P^{-1} T\left(\hat{q}_{n}\right)$ goes to zero. For this we simply choose a real-valued $q_{0} \in C_{0}^{\infty}\left(\mathbb{R}^{2}\right)$ with $\hat{q}_{0}(0)=1$, and set $\hat{q}_{n}=n^{-1} \hat{q}_{0}$. By Theorem 1.1 for any fixed $\chi$ and $\chi_{0}$ the norm of $\Lambda_{\zeta}^{N} A_{1}\left(\hat{q}_{n}, k\right) \Lambda_{\zeta}^{-N}$ as an operator from $C^{\alpha}$ to $C^{\alpha}$ goes to zero as $n \rightarrow \infty$, uniformly for $k \geqq 0, \zeta \in \mathbb{R}^{2}$. Hence, since

$$
h_{1, n}(\xi, \zeta, k)+\left[A_{1}\left(\hat{q}_{n}, k\right) h_{1, n}(\cdot, \zeta, k)\right](\xi)=-\hat{q}_{n}(\xi-\zeta),
$$

it follows that for $n$ sufficiently large,

$$
\operatorname{Re}\left\{h_{1, n}(0,0,0)\right\}<-\frac{1}{2} \hat{q}_{n}(0) \leqq-c\left\|\hat{q}_{n}\right\|_{\alpha, N},
$$

for some $c>0$ independent of $n$, and $\hat{q}_{n} \in \mathcal{O}^{\prime}$. Moreover, since $\hat{q}_{n} \in C_{0}^{\infty}$ the first two estimates of (1.9) hold without the factors of $|\ln k|$. Hence, for $n$ sufficiently large $-\operatorname{Re}\left\{h_{1, n}(0,0,0)\right\} \geqq \varepsilon\left\|h_{1, n}\right\|_{\bar{\alpha}_{, N}}$ for some $\varepsilon>0$ independent of $n$, and $\left\|h_{1, n}\right\|_{\bar{\alpha}_{, N}} \leqq$ $C\left\|\hat{q}_{n}\right\|_{\alpha, N} \leqq c n^{-1}$. Here we use \|\|$_{\alpha, N}$ to denote the $\alpha$-Hölder norm in $(\xi, \zeta, k)$ with weight $\Lambda_{\zeta}^{N}$.

Now we only need to check that the terms in the decomposition of $T P^{-1} T$ which had small norm can now be given small norm uniformly in $n$, and that those that were compact have norm tending to zero as $n \rightarrow \infty$. The choice of $\hat{q}_{n}$ in the preceding paragraph insures that the crucial factor $1-f_{n}(0, k)$ satisfies (see (2.5))

$$
1-f_{n}(0,|\xi|)=1+\left(\frac{h_{1, n}(0,0,0)}{2 \pi}+0\left(\frac{|\xi|^{\alpha}}{n}\right)\right) \ln |\xi|+0\left(\frac{1}{n}\right),
$$

where $\operatorname{Re}\left\{h_{1, n}(0,0,0)\right\} \leqq-\varepsilon n^{-1}, \varepsilon>0$. Hence there is a radius $\rho$ independent of $n$ such that for $n>n_{0}$ and $|\xi|<\rho$,

$$
\frac{\left\|h_{1, n}\right\|_{\alpha, N}}{1-f_{n}(0,|\xi|) \mid} \leqq \frac{C}{n+\varepsilon / 2 \pi|\ln | \xi||} .
$$

The estimate (5.1) shows that the reasoning used to show that the operators $T_{i}\left(\hat{q}_{n}\right)$, $i \neq 0,2,3,5,6,9$ have small norm when cutoff near $\xi=0$ holds uniformly in $n$, and that the reduction of the contributions of $T_{i}, i=2,3,5,6$ to operators of the form (4.17) can be done keeping the norms of the errors uniformly small in $n$. However, one can see that the norms of $T_{0}$ and $T_{9}$ go to zero as $n \rightarrow \infty$ directly from (5.1) and their definitions. We also need to show that $(1-\rho)\left(d S\left(\hat{q}_{n}\right)-P\right)$ goes to zero as $n \rightarrow \infty$. However, since $(1-\rho) S(\hat{q})$ is analytic in $\hat{q}$ at $\hat{q}=0$ and $(1-\rho) P$ is the 
value of its Frechet derivative at $\hat{q}=0$, this is immediate. Finally,

$$
d \beta^{-1}\left(\hat{q}_{n}\right) \dot{\hat{q}}=-\dot{\hat{q}}(0)+R_{n} \dot{\hat{q}},
$$

where $\left\|R_{n}\right\|$ goes to zero as $n \rightarrow \infty$ (see (4.10)). Thus

$$
d S\left(\hat{q}_{n}(\dot{\hat{q}})=d b_{0}\left(\hat{q}_{n}\right) \dot{\hat{q}}+\chi(\xi) d \beta^{-1}\left(\hat{q}_{n}\right) \dot{\hat{q}}=\left(P+T\left(\hat{q}_{n}\right)\right) \dot{\hat{q}},\right.
$$

where $T\left(\hat{q}_{n}\right) P^{-1} T\left(\hat{q}_{n}\right)$ has a small norm in $H_{\alpha, N}$ as $n \rightarrow \infty$. Therefore $d S\left(\hat{q}_{n}\right)$ is invertible large $n$.

As in the proof of Theorem 5.4 of [ER], the analytic dependence of $d S(\hat{q})$ on $\hat{q}$ implies that $\mathscr{U}_{0}$ has a dense open subset $\mathscr{U}_{\infty}$ with $\mathscr{U}_{\infty} \cap H_{\alpha, N}^{r}$ dense in $H_{\alpha, N}^{r}$ such that $d S(\hat{q})$ is invertible for $\hat{q} \in \mathscr{U}_{\infty}$.

Now an application of the implicit function theorem gives

Theorem 5.2. The mapping $\hat{q} \rightarrow S(\hat{q})=b_{0}(\xi)+\beta^{-1} \chi(\xi)$ is a local analytic isomorphism of $H_{\alpha, N}$ at any $\hat{q} \in \mathscr{U}_{\infty}$.

As a final remark we consider, analogously to [ER], the "restricted" backscattering problem. As was noted in [ER], the inverse problem of recovering of a real-valued potential from the backscattering amplitude $b(\xi)$ is still overdetermined since $\hat{q}(\xi)$ satisfies the relation $\hat{q}(\xi)=\overline{\hat{q}(-\xi)}$. Therefore we consider the restricted backscattering problem of recovering a real-valued potential $q(x)$ from

$$
b_{r}(\xi)=\frac{b(\xi)+\overline{b(-\xi)}}{2}
$$

In fact $F^{-1} b_{r}$ is the real part of $F^{-1} b$. Since

$$
b(\xi)=b_{0}(\xi)+\frac{2 \pi}{2 \pi \beta+\ln |\xi|} \chi(\xi)
$$

we have

$$
\begin{aligned}
b_{r}(\xi) & =\frac{b_{0}(\xi)+\overline{b_{0}(-\xi)}}{2}+\frac{1}{2}\left(\frac{2 \pi}{2 \pi \beta+\ln |\xi|}+\frac{2 \pi}{2 \pi \bar{\beta}+\ln |\xi|}\right) \chi(\xi) \\
& =\frac{b_{0}(\xi)+\overline{b_{0}(-\xi)}}{2}+\frac{2 \pi\left(2 \pi \beta_{1}+\ln |\xi|\right)}{\left(2 \pi \beta_{1}+\ln |\xi|\right)^{2}+\left(2 \pi \beta_{2}\right)^{2}} \chi(\xi),
\end{aligned}
$$

where $\beta_{1}=\operatorname{Re} \beta, \beta_{2}=\operatorname{Im} \beta$. Since $b_{r_{0}}(\xi)=\frac{1}{2}\left(b_{0}(\xi)+\overline{b_{0}(-\xi)}\right)=0\left(|\xi|^{\alpha}\right)$ for $|\xi|$ small, knowing $b_{r}(\xi)$ we can recover separately $b_{r_{0}}(\xi), \beta_{1}$ and $\beta_{2}$. Denote by $S_{r}(\hat{q})$ the following map of $H_{\alpha, N}^{r} \cap \mathscr{U}$ to $H_{\alpha N}^{r}$ :

$$
\begin{aligned}
S_{r}(\hat{q}) & =\frac{b_{0}(\xi)+\overline{b_{0}(-\xi)}}{2}+\frac{1}{2}\left(\frac{1}{\beta}+\frac{1}{\bar{\beta}}\right) \chi(\xi) \\
& =b_{r_{0}}(\xi)+\frac{\beta_{1}}{\beta_{1}^{2}+\beta_{2}^{2}} \chi(\xi) .
\end{aligned}
$$

Analogously to the proof of Theorem 5.2 we obtain

Theorem 5.3. $S_{r}(\hat{q})$ is an analytic map of $\mathscr{U} \cap H_{\alpha, N}^{r}$ into $H_{\alpha, N}^{r}$. The Frechet derivative $d S_{r}(\hat{q})$ is a Fredholm operator of index zero for any $\hat{q} \in \mathscr{U} \cap H_{\alpha, N}^{r}$ 
Note that $\mathscr{U} \cap H_{\alpha, N}^{r}$ is not a connected set $\mathscr{H}_{\alpha, N}^{r}$. As in Theorem 5.2 we can prove that $d S_{r}(\hat{q})$ is invertible for some small $\hat{q} \in \mathscr{U} \cap H_{\alpha, N}^{r}$ and this gives that $S_{r}(\hat{q})$ is a local analytic isomorphism for an open dense set in a connected component containing those $\hat{q}$.

In another paper we shall study the invertibility of $d S_{r}(\hat{q})$ in all components of $\mathscr{U} \cap H_{\alpha, N}^{r}$ and also reconsider the same problem for $n=3$.

\section{References}

[BC] Beals, R., Coifman, R.: Multidimensional inverse scattering and nonlinear partial differential equations. Proc. Symp. Pure Math. 43, 45-70. Providence, RI: AMS, 1985

[BML] Bayliss, A., Li, Y., Morawetz, C.: Scattering by potentials using hyperbolic methods. Math. Comp. 52, 321-338 (1989)

[C] Cheney, M.: Inverse scattering in dimension 2. J. Math. Phys. 25, 94-107 (1984)

[ER] Eskin, G., Ralston, J.: The inverse backscattering problem in three dimensions. Commun. Math. Phys. 124, 169-215 (1989)

[F] Faddeev, L. D.: Inverse problem of quantum scattering theory, II. J. Sov. Math. 5, 334-396 (1976)

[M] Melin, A.: The Lippman-Schwinger equation treated as a characteristic Cauchy problem. Seminaire sur EDP 1988-1989, Exposé IV. Ecole Polytech., Palaiseau

[MU] Melrose, R., Uhlmann, G.: Introduction to microlocal analysis with applications to scattering theory, preprint 1989

[NA] Nachman, A., Ablowitz, M.: A multidimensional inverse scattering method. Stud. Appl. Math. 71, 243-250 (1984)

[N] Newton, R.: Inverse scattering II, III, IV. J. Math. Phys. 21, 1968-1715 (1980); J. Math. Phys. 22, 2191-2200 1981 (Correction, J. Math. Phys. 23, 693 (1982)); J. Math. Phys. 23, 594-604 (1982)

[NK] Novikov, R. G., Khenkin, G. M.: The $\bar{\partial}$-equation in multidimensional inverse scattering problems. Russ. Math. Surv. 42, 109-180 (1987)

[P] Prosser, R. J.: Formal solutions of inverse scattering problems I, II, III. J. Math. Phys. 10, 1819-1822 (1969); J. Math. Phys. 17, 1225-1779 (1976); J. Math. Phys. 21, 2648-2653 (1980)

[W] Weder, R.: Generalized limiting absorption method and multidimensional inverse scattering theory. preprint (1990)

Communicated by B. Simon 